\title{
Controls on the distribution of rare earth elements in the Kupferschiefer series of SW Poland
}

\author{
Sławomir OSZCZEPALSKI ${ }^{1, *}$, Andrzej CHMIELEWSKI ${ }^{1}$ and Stanisław Z. MIKULSKI ${ }^{1}$ \\ 1 Polish Geological Institute - National Research Institute, Rakowiecka 4, 00-975 Warszawa, Poland
}

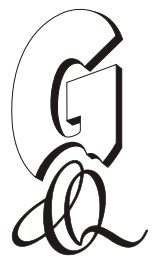

Oszczepalski, S., Chmielewski, A., Mikulski, S.Z., 2016. Controls on the distribution of rare earth elements in the Kupferschiefer series of SW Poland. Geological Quarterly, 60 (4): 811-826, doi: 10.7306/gq.1325

\begin{abstract}
This study deals with the spatial distribution and the PAAS-normalized patterns of rare earth elements (REE) studied by ICP-MS in the Kupferschiefer series of SW Poland. The most characteristic feature is a progressive enrichment in REE content (including $\mathrm{Y}$ and $\mathrm{Sc}$ ) outward from the rocks with pyritic and $\mathrm{Pb}-\mathrm{Zn}$ mineralisation, across the rocks with copper mineralisation, towards the oxidized (Rote Fäule) areas. The same trend can also be observed with respect to LREE, MREE and HREE. Whereas REE distribution diagrams for shales with pyrite and $\mathrm{Pb}-\mathrm{Zn}$ mineralisation are almost flat, diagrams for shales with hematite and copper mineralisation are convex-upward and the strongest convexity is attributed to the transition from hematitic alteration to copper-rich rocks. REE distribution diagrams normalized to PAAS show strong MREE enrichment relative to LREE and HREE, which is typically the highest in the hematite zone, lower in shale with copper mineralisation, and the lowest in shales with $\mathrm{Pb}-\mathrm{Zn}$ and pyrite mineralisation. The systematic increase in the concentration of REE towards the Rote Fäule, and the strong enrichment in MREE in the transition zone are indicative of variable intensity of leaching and formation of the REE enrichments (including MREE) by MREE>HREE>LREE fluids, as evidenced by the MREE-enriched signatures. Overprinting of secondary enrichments over the original REE pattern due to redistribution along the pathways of expanding fluids are postulated to account for the apparent differences in the distribution and concentration of REE (including the MREE-enriched trend) between the oxidized rocks, copper-bearing rocks, and reduced lithologies barren in copper.
\end{abstract}

Key words: rare earth elements, redistribution, Kupferschiefer, Zechstein, SW Poland.

\section{INTRODUCTION}

The Kupferschiefer series (often referred to as the Zechstein copper-bearing series) comprises the lowermost part of the Upper Permian (Wuchiapingian) marine sequence (Zechstein) and the uppermost portion of the terrestrial redbeds (Rotliegend) (Fig. 1). This series is developed as black or dark grey organic-rich and metal sulphide-containing facies ("reduced zone"), and as red-stained organic matter-depleted and iron oxide-bearing rocks ("oxidized zone" = "Rote Fäule"). The Rote Fäule is a zone of post-depositional oxidative alteration exhibiting hematite mineralisation heavily overprinting sulphide minerals (Rydzewski, 1978; Jowett et al., 1987; Oszczepalski, 1989, 1994, 1999; Oszczepalski and Rydzewski, 1991), and features indicative of extensive destruction of organic matter, transformation of clay minerals, and stable isotope variations (Speczik and Püttmann, 1987; Püttmann et al., 1989; Bechtel et al., 1999, 2001a, 2002; Kucha and Przybyłowicz, 1999; Oszczepalski et al., 2002; Speczik et al., 2003). The metals are distinctly zoned not only vertically, but also laterally in belts predominated by cop-

\footnotetext{
* Corresponding author, e-mail: slawomir.oszczepalski@pgi.gov.pl
}

Received: August 5, 2016; accepted: October 14, 2016; first published online: November 2, 2016 per, lead, zinc and pyrite mineralisation, which surround successively the Rote Fäule areas indicating direction of ore fluids flow (Oszczepalski and Rydzewski, 1997; Oszczepalski et al., 2016). The hematite zone forming the inner part of the oxidized area is developed as extensively altered rocks containing sparse relics of sulphides and highly-degraded organic matter. The outermost part of the oxidized zone, which was named as the transition zone between oxidized and reduced rocks (Oszczepalski and Rydzewski, 1991), is dominated by weaker alteration and characterized by replacements of $\mathrm{Cu}$ and $\mathrm{Fe}$ sulphides by iron oxides accompanied by sparsely disseminated remnant copper sulphides and minor glauconites (Oszczepalski, 1994, 1999; Oszczepalski et al., 2002).

General increase in the content of many trace elements (e.g., Se, Re, U and Th) towards the copper zone, and the tendency for certain elements to be depleted (e.g., Co, Ni, Mo, Re, As, Sc, F and Y) or enriched (e.g., Au, PGE, Hg and Sc) in the oxidized Rote Fäule zone have been reported (e.g., Kucha and Przybyłowicz, 1999; Oszczepalski, 1999; Piestrzyński and Wodzicki, 2000; Oszczepalski et al., 2002; Pieczonka et al., 2008; Pieczonka, 2011). Much less attention has been paid to the research of rare earth elements (REE). So far, mostly rock samples from the Lubin-Sieroszowice mining district have been studied (Janczyszyn et al., 1986; Mayer et al., 1992; Sawłowicz, 1994, 2013; Bechtel et al., 2001b; Michalik, 2001). Additional samples from only a few boreholes outside the mining district have been analysed (Mayer et al., 1992; Bechtel et al., 2001b). These studies show that the most enriched in REE are oxidized 


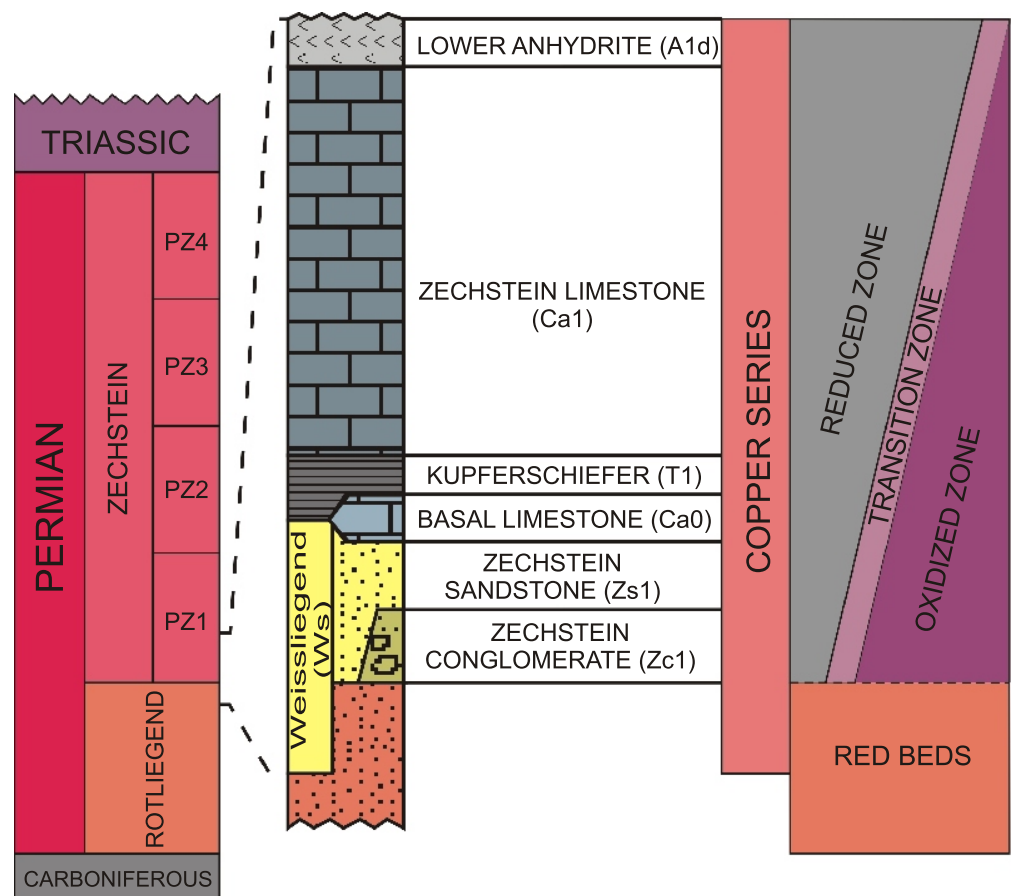

Fig. 1. Stratigraphic position of the Kupferschiefer series and a distribution chart of geochemical zones

Zechstein rocks (Bechtel et al., 2001b; Sawłowicz, 2013) in contrast to reduced facies which does not contain such enrichments, regardless of a presence or absence of rich sulphide mineralisation (Janczyszyn et al., 1986; Mayer et al., 1992; Bechtel et al., 2001b; Sawłowicz, 2013). Therefore, it is extremely vital to examine boreholes from the metal zones that represent different geochemical characteristics and mineralisation patterns throughout SW Poland.

The main goal of this study was to determine the content of REE in the Kupferschiefer series in selected boreholes located in the Sudetic foreland (Fig. 2). Preliminary research into the REE geochemistry described here was carried out at the Polish Geological Institute - National Research Institute (Mikulski et al., 2014). Particular emphasis was placed on the transition zone, occurring in the outermost parts of the oxidized complex, which is considered to be a significant component of the mineralizing system, indicating direction of the alteration trend caused by mineralizing fluids (Oszczepalski and Rydzewski, 1997). In this light, the essential task of this examination was to define the regularities of REE occurrences in the Fore-Sudetic Monocline based on selected boreholes to reveal changes in their content with regard to the oxidized zone (including the transition zone) and zones of sulphide mineralisation. These variations can be used to interpret the factors responsible for REE transport and accumulation. The next aim was to indicate areas with the highest concentrations of rare earth elements. Although REE in the Kupferschiefer series rarely form a considerable concentration, an increasing world demand expects to increase global REE reserves by seeking new sources.

\section{GEOLOGICAL BACKGROUND}

The study area is located within the southwestern part of epi-Variscan platform, which consists of the North Sudetic
Trough and the Fore-Sudetic Monocline, separated by the uplifted Fore-Sudetic Block. These units developed essentially during the Laramide Phase of the Alpine Orogeny as foreland basins to the Sudetes Mountains. The post-Variscan cover of Permian-Mesozoic formations was likely deposited over the Fore-Sudetic Block, but was eroded during Laramide movements. The Permian to Early Triassic extensional stage is consistent with the Late Permian-Early Jurassic tectonic subsidence curves implying that Permian and Triassic rifting predominantly modified the configuration of the Southern Permian Basin (van Wees et al., 2000).

The pre-Permian basement was folded and consolidated during the Late Carboniferous Variscan orogeny. During the Permian, the area of SW Poland belonged to the Southern Permian Basin which was formed at the transition from the Carboniferous to Early Permian as a result of subsidence by rifting (van Wees et al., 2000). A number of sub-depressions separated by ridges developed, including the Wolsztyn-Pogorzela High which separated the Zielona Góra Basin from the Variscan Foreland (Kiersnowski et al., 2010). Rotliegend red-beds accumulated in basins, whereas at ridges the Rotliegend sediments are generally lacking, or the ridges are partly covered by volcanic rocks. The Rotliegend red beds/volcanics are overlain by white fluvial or aeolian sediments forming the Weissliegend sandstones (Fig. 1).

The Zechstein deposition commenced with flooding of the continental Rotliegend basin as a result of rifting-induced subsidence combined with a contemporaneous rise in sea level (Peryt et al., 2010 with references). The topmost parts of the Weissliegend sandstones were reworked by the initial Zechstein transgression, and the transgressive conglomerates and sandstones were locally deposited (mostly in nearshore conditions). With progressive stabilization, the Kupferschiefer was deposited throughout the basin, excluding ridges (Oszczepalski and Rydzewski, 1987). The Kupferschiefer rests above the Zechstein Sandstone or Zechstein Conglomerate, less often above the Basal Limestone, and only sporadically on the Rotliegend siliciclastics and volcanics or pre-Permian basement. The Basal Limestone is present only in shallow-water, nearshore environments. The thickness of the Kupferschiefer varies from several centimetres to $170 \mathrm{~cm}$, with the maximum at depressions and their slopes. Spatial distribution of the lithofacies indicates that the Kupferschiefer was deposited in a low-energy, mud-dominated stratified sea with permanent stagnant bottom-water conditions. Deposition generally took place below the fair-weather wave base. The deep-water facies (organic matter-rich black shales with fine flat lamination) was formed in anaerobic-to-dysaerobic waters, whilst the shallow-water facies (organic matter-containing grey shales consisting mainly of planar- and wavy laminated marlstones and dolomitic shales) formed within the storm wave base in dysaerobic conditions. Variability of the Kupferschiefer sequences is attributed to a fluctuating redoxcline that intersected the submarine topography. The deposition of the Kupferschiefer terminated with an extensive bioturbation and expansion of skeletal fauna indicating that the anaerobic-dysaerobic environment had been replaced by aerobic conditions which are distinctive for the Zechstein Limestone setting. The Zechstein Limestone shows a fairly consistent development throughout the basin, with up to $120 \mathrm{~m}$ of carbonates in nearshore platforms, in contrast to several metres thick carbonates in the basinal settings (Peryt et al., 


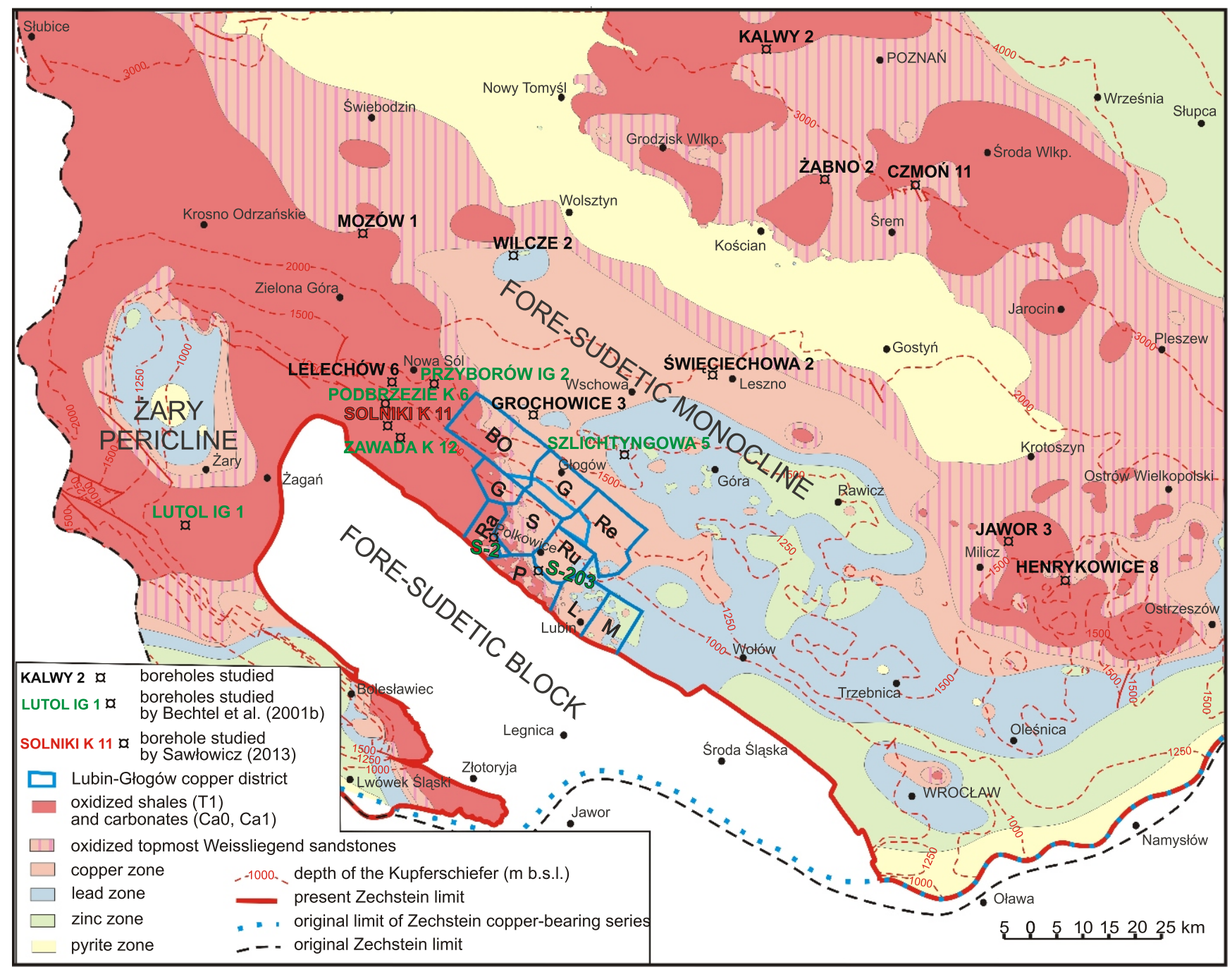

Fig. 2. Map showing location of studied boreholes in relation to the Rote Fäule and metal zoning patterns in the Kupferschiefer series of SW Poland

BO - Bytom Odrzański, G - Głogów, Ga - Gaworzyce, L - Lubin, M - Małomice, P - Polkowice,

$\mathrm{Ra}$ - Radwanice, Re - Retków, Ru - Rudna, S - Sieroszowice

2010, 2012a, b). Most of the intrabasinal palaeohighs are generally lacking both the Rotliegend and Weissliegend sediments and the Kupferschiefer, and are represented by condensed sections (1-5 $\mathrm{m}$ thick) comprising shallow-water carbonates, whereas other ridges are overlain by build-ups up to $90 \mathrm{~m}$ thick (Kiersnowski et al., 2010; Peryt et al., 2012b). The deposition of the Zechstein Limestone was terminated by the Lower Anhydrite which is the lowermost unit of Zechstein evaporites.

The Kupferschiefer mineralisation occurs at the contact between the uppermost Rotliegend redbeds and the Lower Zechstein marine siliciclastics, shales, carbonates and evaporites (Fig. 1). The copper-bearing series comprises the Weissliegend, Basal Limestone, Kupferschiefer and Zechstein Limestone units. Locally, small amounts of sulphides occur at the base of Lower Anhydrite.

The Kupferschiefer-type ore mineralisation rims the Rote Fäule showing a regional-scale metal zonation around the areas of footwall oxidative alteration (Fig. 2). The metals are distributed both vertically and horizontally in the sequence $\mathrm{Fe}^{3+}(\mathrm{Au}, \mathrm{Pt}, \mathrm{Pd})-\mathrm{Cu}(\mathrm{Ag})-\mathrm{Pb}-\mathrm{Zn}-\mathrm{Fe}^{2+}$ in distinct successive zones (Oszczepalski and Rydzewski, 1997). Gold and PGE con- centrated mainly within the Rote Fäule, and particularly in the transition zone (e.g., Piestrzyński et al., 1997; Piestrzyński and Sawłowicz, 1999; Kucha and Przybyłowicz, 1999; Oszczepalski, 1999; Piestrzyński and Wodzicki, 2000; Oszczepalski et al., 2002; Pieczonka et al., 2008). The pyrite zone, characterized by vast predominance of pyrite over $\mathrm{Cu}, \mathrm{Pb}$ and $\mathrm{Zn}$ sulphides, is restricted to the areas most distal from the Rote Fäule, prevailing in marginal parts of the Zechstein basin and in the Wolsztyn-Pogorzela High.

The spatial distribution of metals and the highly significant relationships between copper orebodies and the Rote Fäule areas indicate that the Kupferschiefer mineralizing system developed as a post-sedimentary event caused by a large-scale cross-formational flow of amagmatic hydrothermal fluids from pre-Zechstein footwall rocks during the evolution of the Permian Basin. Substantial flows of metalliferous solutions proceeded within the Rotliegend basin towards palaeohighs, spreading upward and outward, as evidenced by oxidative footwall alteration in feeder areas (Rydzewski, 1978; Oszczepalski and Rydzewski, 1991, 1997; Oszczepalski, 1999). Fluid flows were triggered by convection, compaction, brine recirculation, seismic 
pumping, episodic release or a combination of the processes (Kucha and Pawlikowski, 1986; Jowett et al., 1987; Oszczepalski, 1989, 1999; Cathles et al., 1993; Wodzicki and Piestrzyński, 1994; Blundell et al., 2003). The temperature of mineralizing solutions did not exceed $150^{\circ} \mathrm{C}$ (Oszczepalski, 1999; Oszczepalski et al., 2002; Speczik et al., 2003). It was argued that the large-scale long-lived or multistage fluid flow caused the cross-cutting relationships, expansion of the hematitic alteration front to form the transition zone between the oxidized and ambient reduced rocks, redistribution of metals, and the location of $\mathrm{Cu}-\mathrm{Ag}$ ore bodies directly above and around the noble metals-bearing oxidized rocks. The geochemical and petrologic studies provide evidence for an extensive oxidation of organic matter and pre-existing sulphides as demonstrated by degraded organics and remnant iron and copper sulphides replaced by iron oxides (e.g., Rydzewski, 1978; Speczik and Püttmann, 1987; Püttmann et al., 1989; Oszczepalski and Rydzewski, 1991; Sawłowicz, 1993; Oszczepalski, 1994; Bechtel et al., 2001a, b, 2002; Oszczepalski et al., 2002; Speczik et al., 2003; Chmielewski, 2014; Chmielewski et al., 2015).

\section{PREVIOUS WORK}

The first analytical REE determinations in the lowermost Zechstein sediments of SW Poland were done on 22 samples collected from mines of the Lubin-Sieroszowice district (Janczyszyn et al., 1986). In the initial stage, only La, Eu and Sc (INAA method) were examined. It has been shown that the content of La in the shales varies from 12 to $21 \mathrm{ppm}$, Eu from 0.5 to $1.6 \mathrm{ppm}$, and $\mathrm{Sc}$ in the range of $2-19 \mathrm{ppm}$.
The preliminary investigation in Germany concerning the content of REE (using the INAA method) was performed on six profiles from outcrops in the Sangerhausen region (Hammer et al., 1990). The presented range of REE (La, Ce, Sm, Eu, Tb, Yb and $\mathrm{Lu}$ ) contents show no clear REE concentration patterns in the distribution of REE with respect to mineralisation zones. A weakly expressed trend of slight depletion in La and $\mathrm{Ce}$ is noted only in the copper zone compared with the oxidized and $\mathrm{Zn}-\mathrm{Pb}$ zones. In addition, the study of several samples from the Mansfeld area in Germany (by ICP-MS) revealed that regardless of the presence or absence of rich sulphide mineralisation, lowermost Zechstein rocks are not enriched in REE (Müller et al., 2008).

Broad regional research was conducted by Mayer et al. (1992) who studied 29 samples of mineralized sandstones, shales and dolomites gathered from the Lubin mine, and 25 samples taken from boreholes situated in the eastern part of the Fore-Sudetic Monocline and in the Pomerania region. Based on reduced profiles, the highest REE abundances (INAA method) were measured in the shales, while the lowest content - in the sandstones. According to the authors, this is attributed to an increasing abundance of clay minerals. Distribution diagrams normalized to the NASC standard (North American Shale Composite; cf. Table 1) display the dominance of light REE over heavy REE, negative $\mathrm{Ce}$ and Eu anomalies, and a positive Sm anomaly; nevertheless, the profiles distant from the Lubin-Sieroszowice deposit do not exhibit equivalent regularities.

Further studies of REE concentrations (ICP-MS method) in shales were done on seven samples from boreholes located in the vicinity of the Fore-Sudetic Block (Fig. 2), and two samples from the Pomerania region in order to determine variability in REE concentration and distribution with respect to mineralisation zones (Bechtel et al., 2001b). For this purpose, samples

Ta ble 1

Average REE composition (ppm) of rock standards

\begin{tabular}{|l|c|c|c|c|c|c|c|c|}
\hline \multicolumn{2}{|c|}{ REE } & Chondrite & $\begin{array}{c}\text { Upper } \\
\text { Continental Crust }\end{array}$ & $\begin{array}{c}\text { World Shale } \\
\text { Average }\end{array}$ & NASC & PAAS & $\begin{array}{c}\text { SDO-1 } \\
\text { Black Shales }\end{array}$ & $\begin{array}{c}\text { SDO-1 } \\
\text { Metalliferous } \\
\text { Black Shales }\end{array}$ \\
\hline Lanthanum & La & 0.237 & 31 & 41.00 & 31.1 & 38.2 & 38.5 & 77.0 \\
\hline Cerium & Ce & 0.613 & 63 & 83.00 & 66.7 & 79.6 & 79.3 & 158.6 \\
\hline Prazeodymium & $\mathrm{Pr}$ & 0.0928 & 7.1 & 10.10 & 7.70 & 8.83 & 8.9 & 19.8 \\
\hline Neodymium & $\mathrm{Nd}$ & 0.457 & 27 & 38.00 & 27.4 & 33.9 & 36.6 & 73.2 \\
\hline Samarium & $\mathrm{Sm}$ & 0.148 & 4.7 & 7.50 & 5.59 & 5.55 & 7.7 & 15.4 \\
\hline Europium & $\mathrm{Eu}$ & 0.0563 & 1.0 & 1.61 & 1.18 & 1.08 & 1.6 & 3.2 \\
\hline Gadolinium & $\mathrm{Gd}$ & 0.199 & 4.0 & 6.35 & 4.90 & 4.66 & 6.5 & 13.0 \\
\hline Terbium & $\mathrm{Tb}$ & 0.0361 & 0.7 & 1.23 & 0.85 & 0.774 & 1.2 & 2.4 \\
\hline Dysprosium & $\mathrm{Dy}$ & 0.246 & 3.9 & 5.50 & 4.17 & 4.68 & 6.0 & 11.4 \\
\hline Holmium & $\mathrm{Ho}$ & 0.0546 & 0.83 & 1.34 & 1.02 & 0.991 & 1.2 & 0.2 \\
\hline Erbium & $\mathrm{Er}$ & 0.160 & 2.3 & 3.75 & 2.84 & 2.85 & 3.6 & 7.2 \\
\hline Thulium & $\mathrm{Tm}$ & 0.0247 & 0.3 & 0.63 & 0.48 & 0.405 & 0.45 & 0.9 \\
\hline Ytterbium & $\mathrm{Yb}$ & 0.161 & 2.0 & 3.53 & 3.06 & 2.82 & 3.4 & 6.8 \\
\hline Lutetium & $\mathrm{Lu}$ & 0.0246 & 0.31 & 0.61 & 0.46 & 0.433 & 0.54 & 1.0 \\
\hline SREE & & 2.5 & 148.1 & 204.2 & 157.5 & 184.8 & 195.5 & 390.1 \\
\hline Yttrium & $\mathrm{Y}$ & 1.57 & 21 & & 35 & 27 & 40.6 & 81.2 \\
\hline Scandium & $\mathrm{Sc}$ & & 14 & & 14.9 & 16 & 13.2 & 26.4 \\
\hline
\end{tabular}

Chondrite (McDonough and Sun, 1995), Upper Continental Crust (Rudnick and Gao, 2003), World Shale Average (Piper, 1974), NASC - North American Shale Composite (Gromet et al., 1984), PAAS - Post-Archean Australian Shale (McLennan, 1989), SDO-1 - Black Shales (Huyck, 1989), SDO-1 - Metalliferous Black Shales (Huyck, 1989) 
from the hematite, $\mathrm{Cu}, \mathrm{Zn}-\mathrm{Pb}$, and pyrite zones were examined. The highest contents of REE were found in oxidized shales (in the range of 99 to $211 \mathrm{ppm}$, average $142 \mathrm{ppm}$ ), lower concentration in shales with $\mathrm{Cu}, \mathrm{Pb}$ and $\mathrm{Zn}$ mineralisation (69-96 ppm; average $83 \mathrm{ppm}$ ), and the lowest concentration in pyritic shales (60-65 ppm; average 62 ppm). The weakly oxidized shales from the transition zone are characterized by a significantly higher average content of REE (211 ppm) than the strongly oxidized shales (125 ppm). The highest REE concentration occurs in the sample from the transition zone $(210.81 \mathrm{ppm}$ in the S-2 borehole). Diagrams normalized to both chondrite and ES (European Shale Composite) show strong enrichment in MREE ( $\mathrm{Nd}, \mathrm{Sm}, \mathrm{Eu}$ and $\mathrm{Gd}$ ) compared with LREE (La and $\mathrm{Ce}$ ) and HREE (Dy, Er, Yb and Lu). Oxidized rocks have a positive anomaly for Sm and negative $\mathrm{Ce}$ and $\mathrm{Eu}$ anomalies. Summing up, a gradual decrease in REE concentration (including LREE, MREE and HREE) was observed starting from oxidized shales (142 ppm) through copper shales (83 ppm) to shales with pyrite mineralisation located far away from copper deposits (62 ppm), as well as when going from highly oxidized (125 ppm) to poorly oxidized shales (211 ppm).

Based on 32 samples from the Lubin-Sieroszowice district, Michalik (2001) reported the highest concentration of REE in the uppermost Weissliegend, linking this enrichment with the presence of carbonate cements, phosphates, fluorides, and Fe-oxide rims around detrital grains.

Sawłowicz $(1994,2013)$ achieved comparable results to those presented by Bechtel et al. (2001b) by the study of rock samples from the Lubin-Sieroszowice district (ICP-MS and INAA). This study demonstrates the overall REE budget in different regions and seven REE diagrams for selected samples of sandstones, shales and dolomites normalized to the NASC standard. In addition, plots for 11 samples with copper mineralisation, two samples with $\mathrm{Pb}-\mathrm{Zn}$ mineralisation and 10 oxidized samples (including six samples from the transition zone) are presented. It has been shown that the largest amounts of REE (including MREE, $\mathrm{Y}$ and Sc) are in the oxidized profiles (average REE concentration range 60-298 ppm, in the Polkowice Mine and in the Solniki K 11 borehole), lower concentrations are recorded in the profiles with copper mineralisation (117-158 ppm) and zinc-lead-bearing profiles (126-160 ppm). The oxidized samples have elevated concentrations of Sc (13-19 ppm) and $Y(8-45 \mathrm{ppm})$ compared to reduced samples (7-12, 7-27 ppm, respectively), and are enriched in MREE, as demonstrated by the REE convex-upwards distribution patterns. REE concentration values in dolomites and sandstones are distinctly lower than in shales (average contents are 73 and $82 \mathrm{ppm}$, respectively).

Accepting the mineralizing system, in which the formation of the Rote Fäule/ore system resulted from the upward and lateral advancing alteration by hydrothermal solutions, it is believed that enrichment of REE resulted not only from the delivery by mineralizing fluids but primarily from remobilization of REE from the altering lowermost Zechstein bedrock, and their redistribution during the subsequent flow. Bechtel et al. (2001b) stated that REE enrichments (including MREE) in oxidized lithologies are the result of secondary supply of REE by fluids of MREE > HREE > LREE type and their subsequent deposition at the redox front. Alternatively, REE may have been remobilized from originally reduced profiles by oxidizing solutions responsible for the Rote Fäule alteration, during the formation of neomorphic illite, iron oxides or phosphates (cf. Bechtel et al.,
1999, 2001a, b; Michalik, 2001; Oszczepalski et al., 2002). Sawłowicz (2013) have shown that the largest amount of REE (including MREE) and $Y$ and Sc were accumulated in the oxidized part of profiles. Unlike Bechtel et al. (2001b), Sawłowicz (2013) assumed that there are no significant differences in the distributions of REE between strongly oxidized samples and samples from the transition zone. However, the reported REE distribution data unequivocally display that oxidized samples have considerable contents of REE in the range of 60-298 ppm, with the highest values in samples from the transition zone (188-298 ppm) and lower values for extensively oxidized samples (60-269 ppm). Both types of samples (heavily oxidized and poorly oxidized) are enriched in REE (average $247 \mathrm{ppm}$ ) and lacking in Eu anomalies in comparison with copper-rich samples. On the basis of variations in REE distributions and their enrichments in oxidized and copper-bearing profiles, Sawłowicz (2013) supports the view of Bechtel et al. (2001b) that this enrichment is due to the upward influx of mineralizing solutions from Rotliegend units and believes that differences in REE concentrations in oxidized and copper-bearing profiles are caused by a higher flow rate and (or) total volume of mineralizing solutions in oxidized areas than in the copper zone. In contrast to previous studies (e.g., Bechtel et al., 1999, 2001b; Oszczepalski, 1999), Sawłowicz (2013) suggested that oxidation of copper sulphides and their removal were related to the later action of mineralizing or post-mineralizing solutions and that late-stage fluids could be oxidizing but not mineralising.

\section{SAMPLING AND ANALYTICAL METHODS}

A representative suite of 26 samples from 10 boreholes located in the Fore-Sudetic Monocline (outside the Lubin-Sieroszowice copper district) was collected from all lithostratigraphic units of the Kupferschiefer series (Fig. 1). An additional sample from the Zdrada IG 8 borehole located in northern Poland was also studied. The examination covered 14 shale samples collected from the Kupferschiefer, nine Weissliegend sandstone samples, two samples from the Zechstein Limestone and two other specimens from the Basal Limestone. Thirteen samples represent reduced facies and 14 samples are from the oxidized zone (Appendix $1^{*}$ ). Among samples of oxidized rocks, eight come from the strongly oxidized part of the profiles and six samples from the slightly oxidized transition zone, whereas samples of reduced rocks comprise copper, $\mathrm{Pb}-\mathrm{Zn}$ and pyrite mineralisation patterns.

The REE ICP-MS analyses were performed at the Chemical Laboratory of the Polish Geological Institute - National Research Institute, using Elan DRC II Perkin Elmer mass spectrometry. The calibration of external standards were prepared from multi-element stock solutions from AccuStandard. After acid digestion, the samples were diluted 20 times with a solution of $1 \% \mathrm{HNO}_{3}$. Total solution of elements contained in the samples was achieved by use of hydrofluoric acid and chloric acid (VII - oxidation of fluorine and chlorine in acid). Dried and powdered samples were initially treated with nitric acid $(\mathrm{V})$ in order to distribute organic matter and dissolved in a mixture of perchloric and hydrofluoric acid (VII). In order to improve the procedure for dissolution, the digestion process was repeated three times. In the last stage, after evaporation to dryness (on a hot plate, to a temperature of $150 \pm 10^{\circ} \mathrm{C}$ ) the residue was dissolved in dilute nitric acid (V). The solution obtained was sub-

* Supplementary data associated with this article can be found, in the online version, at doi: 10.7306/gq.1325 
mitted to ICP-MS analysis enabling achievement of low detection limits of $0.5 \mathrm{ppm}$ for LREE and $0.05 \mathrm{ppm}$ for HREE. Abundances of base metals were determined by the XRF method, and Au, Pt, and Pd by ET AAS.

\section{RESULTS}

\section{ANALYTICAL DATA}

In order to identify changes in REE variation and distribution, Kupferschiefer and Weissliegend samples were grouped with respect to geochemical zones into oxidized and reduced samples and into rocks characterized by hematite, copper, $\mathrm{Pb}-\mathrm{Zn}$, and pyrite mineralisation, considering zones of respective mineralisation (Appendix 1). Since the number of carbonate samples is insignificant, the following studies of REE abundances were based mainly on shale and sandstone units. The concentrations of selected base metals are presented in Appendix 1 and the total and average contents of REE, $\mathrm{Y}$ and $\mathrm{Sc}$ for all samples are presented in Appendix 2. Average REE contents in the Kupferschiefer and Weissliegend samples grouped with respect to redox and mineralisation zones have been calculated (Table 2).

Due to significant differences in concentrations of individual REE, as proposed by Sholkovitz (1995) and followed by Bechtel et al. (2001b), rare earth elements were divided into three groups comprising light REE (LREE): La, Ce, middle REE (MREE): Nd, Sm, Eu, Gd, and heavy REE (HREE): Dy, $\mathrm{Ho}, \mathrm{Er}, \mathrm{Tm}, \mathrm{Yb}$ and Lu.

To interpret variations in REE abundances in studied samples, different standards are typically used (Table 1), however, the normalization of REE concentrations against the chondrite and PAAS (post-Archean Australian Shale, cf. Table 1) standards are now more commonly used (McLennan, 1989; McDonough and Sun, 1995). A Kupferschiefer sample from the Zdrada IG 8 borehole with pyritic mineralisation, barren of $\mathrm{Cu}, \mathrm{Pb}$ and $\mathrm{Zn}$ (Appendices 1 and 2), is also significant for comparisons. It was recently characterized by Peryt et al. (2012a). The REE content in this sample is similar to REE concentrations in black shale standards without rich sulphide mineralisation (cf. Table 1), showing the background distribution of REE.

The REE distributions in Kupferschiefer samples are presented as diagrams, both chondrite-normalized (Fig. 3) and PAAS-normalized patterns (Figs. 4-8), whereas the distribution of REE in Weissliegend samples is normalized to chondrite (Fig. 9). To show enrichments of some REE relative to other elements and with respect to the redox zones (reduced vs. oxidized) and zones of mineralisation (hematitic, copper, $\mathrm{Pb}-\mathrm{Zn}$, and pyrite), PAAS-normalized mean values and related parameters are presented (Tables 2 and 3).

\section{REE VARIATIONS IN LITHOLOGIES}

According to the acquired data, the distribution and content of REE vary significantly depending on lithology (Appendix 2). The highest $\Sigma$ REE concentrations are recorded in the Kupferschiefer (115-318 ppm, average 201 ppm) and considerably lower concentrations are in carbonates of the Zechstein Limestone and Basal Limestone (50-154 ppm, average $95 \mathrm{ppm}$ ), whereas the lowest values are typical for Weissliegend sandstone samples (42-186 ppm, average $87 \mathrm{ppm}$ ). This trend is clearly visible in the chondrite-normal-

$\sim$
$\frac{0}{0}$
$\stackrel{0}{\vdash}$

\begin{tabular}{|c|c|c|c|c|c|c|c|c|c|c|c|c|c|}
\hline ᄃ & $\lambda$ & 0 & $\Lambda$ & $\nabla$ & $N$ & - & $\nabla$ & $m$ & $\begin{array}{lll} & L\end{array}$ & L) & $\nabla$ & $N$ & $m$ \\
\hline œ & 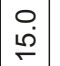 & $\begin{array}{l}\infty \\
\infty \\
\infty\end{array}$ & 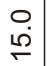 & $\stackrel{\circ}{\circ}$ & $\stackrel{+}{\infty}$ & $\stackrel{\nabla}{\sharp}$ & \begin{tabular}{|l|}
$L$ \\
$\dot{J}$ \\
\end{tabular} & 菅 & $\begin{array}{ll}\infty & 1 \\
m & 1\end{array}$ & $\hat{\sim}$ & $\begin{array}{l}\infty \\
\dot{m}\end{array}$ & $\stackrel{m}{\sim}$ & $\stackrel{\leftrightarrow}{N}$ \\
\hline 壳 & 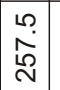 & $\begin{array}{l}\infty \\
i \infty \\
\infty \\
\infty \\
\end{array}$ & 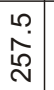 & $\begin{array}{l}\circ \\
\dot{0} \\
\circ \\
\end{array}$ & 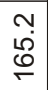 & $\begin{array}{l}\stackrel{\sim}{N} \\
\stackrel{2}{N} \\
\end{array}$ & $\overline{\grave{N}}$ & $\begin{array}{l}0 \\
\tilde{m} \\
\dot{m}\end{array}$ & $\begin{array}{ll}r \\
m \\
m\end{array}$ & 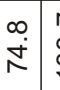 & $\begin{array}{l}\stackrel{M}{m} \\
\stackrel{m}{\sigma}\end{array}$ & $\begin{array}{l}0 \\
\infty \\
\infty\end{array}$ & $\overline{0}$ \\
\hline$>$ & \begin{tabular}{l|}
0 \\
$\dot{j}$ \\
$\tilde{m}$
\end{tabular} & $\underset{N}{N}$ & $\begin{array}{l}0 \\
\dot{m}\end{array}$ & $\stackrel{\check{J}}{\sim}$ & $\begin{array}{l}m \\
\infty \\
\sim\end{array}$ & $\stackrel{r}{\check{r}}$ & $\begin{array}{l}0 \\
\infty \\
\stackrel{\infty}{\sim}\end{array}$ & $\hat{\stackrel{\Im}{\Im}}$ & $\begin{array}{l}\hat{\sigma} \\
\stackrel{2}{\sigma}\end{array}$ & $\begin{array}{l}\circ \\
\stackrel{\circ}{\circ}\end{array}$ & $\hat{\stackrel{\sigma}{\sigma}}$ & $\stackrel{\stackrel{N}{N}}{\sim}$ & $\begin{array}{l}\bullet \\
\infty\end{array}$ \\
\hline 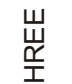 & 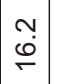 & $\stackrel{\circ}{\circ}$ & 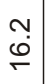 & 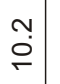 & $\begin{array}{l}0 \\
\infty \\
\infty\end{array}$ & $\stackrel{\bullet}{\Gamma}$ & $\begin{array}{l}\stackrel{0}{m} \\
\stackrel{m}{r}\end{array}$ & $\stackrel{\nabla}{\grave{N}}$ & $\hat{\sim}$ & $\stackrel{ナ}{\triangleright}$ & $\hat{\imath}$ & $\begin{array}{c}N \\
\text { No }\end{array}$ & 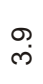 \\
\hline 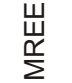 & \begin{tabular}{|l|}
0 \\
$\infty$ \\
0 \\
0
\end{tabular} & $\begin{array}{l}\circ \\
\stackrel{g}{\sigma}\end{array}$ & $\begin{array}{l}0 \\
\infty \\
\infty \\
0\end{array}$ & $\check{\tilde{D}}$ & $\begin{array}{l}0 \\
\dot{0} \\
\dot{q}\end{array}$ & $\begin{array}{l}\forall \\
\stackrel{\sigma}{\sigma}\end{array}$ & $\begin{array}{l}10 \\
0 \\
0\end{array}$ & $\begin{array}{l}\infty \\
\stackrel{\infty}{\infty} \\
\infty \\
\infty\end{array}$ & $\begin{array}{cc}N \\
\vdots \\
o \\
⿱ 亠 乂\end{array}$ & $\stackrel{\circ}{\grave{N}}$ & $\begin{array}{c}\text { No } \\
\text { Oे }\end{array}$ & 吕 & $\begin{array}{l}m \\
\infty \\
\infty\end{array}$ \\
\hline 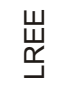 & $\stackrel{-}{\stackrel{i}{\sim}}$ & $\hat{\stackrel{o}{\circ}}$ & $\underset{\stackrel{\leftarrow}{S}}{\stackrel{D}{\sim}}$ & $\begin{array}{l}\hat{\infty} \\
\infty\end{array}$ & $\begin{array}{l}\text { ळ } \\
\varnothing\end{array}$ & $\begin{array}{l}\mathscr{\sigma} \\
\ddot{\rho}\end{array}$ & $\underset{⿱ 亠}{\check{r}}$ & 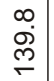 & $\check{8}$ & $\begin{array}{l}\dot{\varphi} \\
\stackrel{\rho}{\rho}\end{array}$ & চ্் & 官 & $\frac{\dot{m}}{m}$ \\
\hline$\underset{\widetilde{\Psi}}{\breve{\Psi}}$ & $\begin{array}{l}0 \\
\stackrel{N}{N} \\
N\end{array}$ & $\begin{array}{l}10 \\
\\
0 \\
0\end{array}$ & ㅇ. & $\begin{array}{l}\infty \\
\stackrel{\infty}{\Gamma}\end{array}$ & $\begin{array}{l}\infty \\
\dot{v} \\
\dot{\sigma}\end{array}$ & 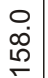 & 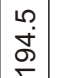 & 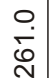 & $\begin{array}{l}0 \\
\dot{T} \\
\tau\end{array}$ & $\begin{array}{l}\infty \\
\dot{0} \\
\text { (1) }\end{array}$ & 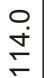 & \begin{tabular}{|l|}
$\infty$ \\
0 \\
0
\end{tabular} & $\begin{array}{l}\infty \\
0 \\
i \\
\llcorner\end{array}$ \\
\hline כ & $\begin{array}{c}\bar{\tau} \\
\dot{\sigma}\end{array}$ & $\begin{array}{l}\stackrel{\sim}{\sim} \\
\text { ஸे }\end{array}$ & $\underset{\tau}{\check{\sigma}}$ & $\underset{\sim}{\tilde{O}}$ & $\underset{ָ}{\tilde{O}}$ & 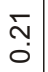 & \begin{tabular}{c|}
$\infty$ \\
$m$ \\
0 \\
0
\end{tabular} & 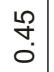 & $\frac{n}{\sigma}$ & $\stackrel{1}{\check{0}}$ & $\stackrel{N}{\check{0}}$ & $\stackrel{\nabla}{\square}$ & 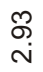 \\
\hline$\stackrel{0}{2}$ & $\begin{array}{l}0 \\
\infty \\
\end{array}$ & $\begin{array}{l}\stackrel{N}{N} \\
\stackrel{r}{r}\end{array}$ & $\begin{array}{l}\infty \\
\infty \\
i\end{array}$ & 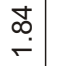 & 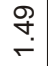 & $\underset{\forall}{\stackrel{\nabla}{\leftarrow}}$ & $\begin{array}{l}L \\
\omega \\
\mathrm{N} \\
\mathrm{N}\end{array}$ & $\begin{array}{l}\stackrel{\infty}{N} \\
m\end{array}$ & $\underset{ָ ָ}{\sim}$ & $\begin{array}{l}0 \\
\infty \\
0 \\
0\end{array}$ & 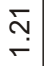 & \begin{tabular}{l}
\multirow{2}{*}{} \\
0 \\
0
\end{tabular} & $\begin{array}{l}\pi \\
0\end{array}$ \\
\hline$\varepsilon$ & \begin{tabular}{l}
$\infty$ \\
\multirow{1}{*}{} \\
0
\end{tabular} & $\begin{array}{c}\stackrel{\infty}{N} \\
\stackrel{0}{0}\end{array}$ & 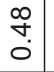 & $\begin{array}{l}\text { లి } \\
0\end{array}$ & $\stackrel{\stackrel{L}{N}}{0}$ & 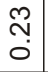 & $\begin{array}{l}\tau \\
\dot{t} \\
0\end{array}$ & $\begin{array}{l}\hat{n} \\
0 \\
0\end{array}$ & 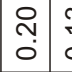 & $\frac{m}{\check{0}}$ & 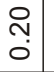 & $\frac{0}{0}$ & $\check{\check{\sigma}}$ \\
\hline யั & \begin{tabular}{|c|}
$\bar{\sigma}$ \\
$\dot{m}$
\end{tabular} & $\begin{array}{l}\text { D } \\
\text { i }\end{array}$ & \begin{tabular}{c|}
$\bar{\sigma}$ \\
$\dot{m}$
\end{tabular} & 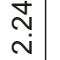 & $\stackrel{\infty}{\stackrel{\infty}{r}}$ & $\widehat{\hat{\sigma}}$ & 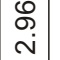 & $\begin{array}{l}\hat{f} \\
\dot{\sigma}\end{array}$ & đִ & \begin{tabular}{l|l}
$\mathscr{O}$ \\
$\stackrel{\circ}{\circ}$ \\
$\circ$
\end{tabular} & ֶָ. & $\stackrel{⿱}{\check{\leftarrow}}$ & $\begin{array}{l}m \\
\infty \\
0 \\
0\end{array}$ \\
\hline 우 & 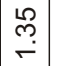 & $\begin{array}{l}9 \\
T \\
0\end{array}$ & 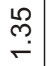 & $\begin{array}{l}\llcorner \\
\infty \\
0 \\
0\end{array}$ & $\begin{array}{l}\hat{\omega} \\
\dot{0}\end{array}$ & $\begin{array}{l}\tilde{0} \\
0 \\
0\end{array}$ & $\stackrel{0}{\stackrel{0}{0}}$ & $\stackrel{r}{\stackrel{5}{\pi}}$ & $\begin{array}{ll}0 & 1 \\
0 & 1 \\
0 & 1\end{array}$ & $\begin{array}{c}\hat{m} \\
0\end{array}$ & $\begin{array}{l}\mathscr{0} \\
\stackrel{0}{0}\end{array}$ & \begin{tabular}{l}
\multirow{2}{*}{} \\
$\dot{0}$
\end{tabular} & లె \\
\hline$\widehat{\Delta}$ & \begin{tabular}{|l|} 
\\
\multirow{2}{*}{} \\
\end{tabular} & $\begin{array}{l}\text { Ðे } \\
\dot{\nabla}\end{array}$ & 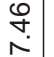 & $\begin{array}{l}\bar{N} \\
\dot{\nabla}\end{array}$ & $\begin{array}{l}\stackrel{0}{0} \\
\ddot{m}\end{array}$ & $\begin{array}{l}\stackrel{o}{+} \\
\text { m. }\end{array}$ & $\begin{array}{l} \\
0 \\
0 \\
0\end{array}$ & $\begin{array}{l}\infty \\
\infty \\
\infty \\
\infty\end{array}$ & $\begin{array}{ll}\text { Oे } & \\
\text { m }\end{array}$ & $\begin{array}{c}\hat{O} \\
\stackrel{N}{*}\end{array}$ & $\begin{array}{l}\text { ब. } \\
\text { ले }\end{array}$ & 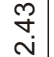 & $\begin{array}{c}m \\
\infty \\
\sim \\
-\end{array}$ \\
\hline$\stackrel{\rho}{F}$ & $\stackrel{n}{m}$ & \begin{tabular}{l}
\multirow{0}{*}{} \\
$\infty$ \\
0
\end{tabular} & 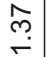 & శ్ & $\begin{array}{l}\infty \\
0 \\
0 \\
0\end{array}$ & $\begin{array}{l}\mathscr{0} \\
\ddot{0} \\
\dot{0}\end{array}$ & 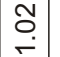 & 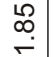 & \begin{tabular}{l|l}
9 & 5 \\
0 & 0
\end{tabular} & \begin{tabular}{l|l}
$\circ$ & 1 \\
\multirow{0}{*}{} & 1
\end{tabular} & $\begin{array}{l}2 \\
2 \\
0\end{array}$ & $\begin{array}{l}0 \\
\dot{0} \\
0\end{array}$ & $\begin{array}{l}0 \\
\text { m. } \\
0\end{array}$ \\
\hline 이 & $\mid \begin{array}{l}m \\
\vdots \\
\sigma\end{array}$ & $\frac{10}{\leftarrow 0}$ & $\frac{m}{\sigma}$ & $\begin{array}{l}\circ \\
\infty \\
0\end{array}$ & $\begin{array}{l}\mathscr{Q} \\
\infty \\
\dot{\sigma}\end{array}$ & $\begin{array}{l}\hat{\infty} \\
\dot{\sigma}\end{array}$ & $\mid \begin{array}{l}m \\
0 \\
0\end{array}$ & $\begin{array}{l}\infty \\
\stackrel{\infty}{\sim} \\
\stackrel{1}{\simeq}\end{array}$ & \begin{tabular}{l|l}
0 & \\
2 & $\delta$ \\
$\omega$ &
\end{tabular} & \begin{tabular}{l|l}
$\infty$ \\
$\infty$ \\
\end{tabular} & $\begin{array}{l}0 \\
2 \\
i \\
0\end{array}$ & $\underset{\sim}{\stackrel{\sim}{n}}$ & $\begin{array}{l}\stackrel{8}{0} \\
\text { in }\end{array}$ \\
\hline$\vec{山}$ & $\frac{0}{i}$ & | & $\stackrel{\sigma}{i}$ & 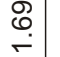 & 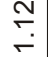 & 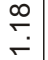 & 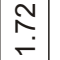 & $\begin{array}{l}\infty \\
\infty \\
i\end{array}$ & re & \begin{tabular}{l}
\multirow{N}{*}{} \\
0
\end{tabular} & 怘 & $\mid \begin{array}{l}\infty \\
\stackrel{1}{0} \\
0\end{array}$ & 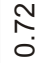 \\
\hline$\frac{\varepsilon}{\omega}$ & 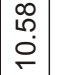 & 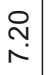 & $\begin{array}{l}\infty \\
\stackrel{\infty}{0} \\
0 \\
0\end{array}$ & $\stackrel{\circledast}{\curvearrowright}$ & $\begin{array}{l}\mathfrak{N} \\
i \infty\end{array}$ & $\begin{array}{l}\hat{0} \\
0\end{array}$ & $\mid \begin{array}{c}\tilde{N} \\
\infty \\
\infty\end{array}$ & 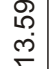 & 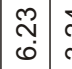 & \begin{tabular}{c|c}
$\stackrel{\sim}{N}$ \\
$\oplus$
\end{tabular} & $\begin{array}{l}\stackrel{m}{*} \\
0\end{array}$ & $\begin{array}{l}0 \\
i \\
m\end{array} \mid$ & @ু \\
\hline 주 & \begin{tabular}{l}
0 \\
\multirow{2}{*}{} \\
\multirow{2}{*}{}
\end{tabular} & $\begin{array}{l}\text { N } \\
\text { ले }\end{array}$ & \begin{tabular}{l|}
$\stackrel{0}{+}$ \\
$\stackrel{f}{f}$
\end{tabular} & $\begin{array}{l}\hat{\dot{e}} \\
\dot{e}\end{array}$ & ָั & $\stackrel{m}{m}$ & $\mid \begin{array}{l}10 \\
\rho \\
\rho \\
ల\end{array}$ & 官 & $\begin{array}{l}\hat{\omega} \\
\hat{N}\end{array}$ & $\underset{+}{\check{J}}$ & $\hat{\grave{N}}$ & $\stackrel{m}{\stackrel{m}{\sim}}$ & $\stackrel{O}{\stackrel{O}{N}}$ \\
\hline$\overline{0}$ & $\stackrel{+}{\check{\Sigma}}$ & $\begin{array}{l}+ \\
\infty\end{array}$ & $\stackrel{⿱ 亠 凶}{\leftarrow}$ & $\begin{array}{l}\infty \\
\infty\end{array}$ & $\stackrel{⿱}{\sim}$ & $\begin{array}{l}\infty \\
\infty \\
\infty\end{array}$ & $\begin{array}{l}\sigma \\
\sigma \\
\sigma\end{array}$ & $\stackrel{\vec{m}}{\stackrel{m}{\leftarrow}}$ & $\check{0}$ & $\begin{array}{l}\stackrel{\bullet}{\oplus} \\
\dot{m}\end{array}$ & $\check{\sigma}$ & $\underset{\sim}{\sim}$ & $\stackrel{\leftrightarrow}{\sim}$ \\
\hline$\bigcup^{0}$ & $\mid \begin{array}{l}10 \\
10 \\
\infty\end{array}$ & $\begin{array}{l}\text { ๑ } \\
\dot{0}\end{array}$ & $\begin{array}{l}10 \\
10 \\
\infty \\
\infty\end{array}$ & กొ & $\begin{array}{l}6 \\
\stackrel{0}{ }\end{array}$ & 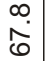 & $\mid \begin{array}{l}10 \\
0 \\
0\end{array}$ & 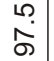 & $\stackrel{m}{\dot{\gamma}}$ & $\begin{array}{l}\stackrel{+}{+} \\
\stackrel{+}{N}\end{array}$ & $\frac{m}{\dot{q}}$ & $\begin{array}{l}N \\
\stackrel{N}{N} \\
\stackrel{2}{*}\end{array}$ & $\stackrel{\check{\Sigma}}{\grave{N}}$ \\
\hline$\stackrel{\widetilde{\top}}{\mathrm{T}}$ & \begin{tabular}{|l|} 
\\
\\
\end{tabular} & $\begin{array}{l}\text { @ } \\
\grave{ల}\end{array}$ & $\begin{array}{l}\dot{0} \\
\ddot{j}\end{array}$ & ஸ̣ & $\begin{array}{l}n \\
\dot{p}\end{array}$ & $\check{c}$ & $\begin{array}{l}0 \\
\hat{m} \\
\end{array}$ & 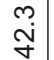 & $\begin{array}{ll}\infty & 0 \\
& \mathbf{n}\end{array}$ & $\begin{array}{ll}\stackrel{N}{\mp} & 1\end{array}$ & $\begin{array}{l}\infty \\
\stackrel{\infty}{-}\end{array}$ & 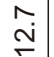 & 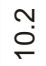 \\
\hline 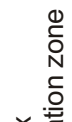 & 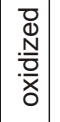 & 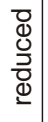 & 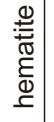 & $\begin{array}{l}\bar{\Phi} \\
\overline{0} \\
\overline{0} \\
0\end{array}$ & $\begin{array}{l}\text { N } \\
\frac{1}{\alpha} \\
0.5\end{array}$ & 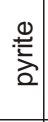 & 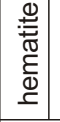 & 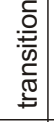 & 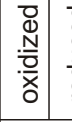 & $\begin{array}{l}\bar{d} \\
0 \\
\underline{0} \\
\underline{\Phi} \\
\underline{d}\end{array}$ & 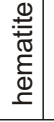 & \begin{tabular}{|l|}
$\overline{\bar{\alpha}}$ \\
$\stackrel{0}{0}$ \\
\\
\end{tabular} & $\begin{array}{l}\text { N్ } \\
\text { ô } \\
\alpha\end{array}$ \\
\hline 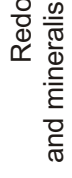 & $\begin{array}{l}x \\
\frac{0}{0} \\
d \\
d\end{array}$ & & & & s & & 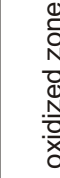 & 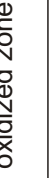 & 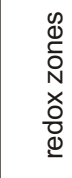 & & & 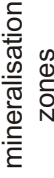 & \\
\hline 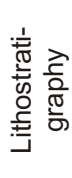 & \multicolumn{8}{|c|}{ 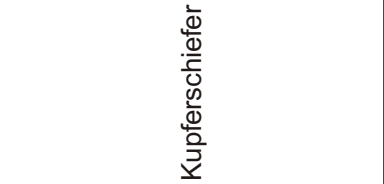 } & \multicolumn{5}{|c|}{ 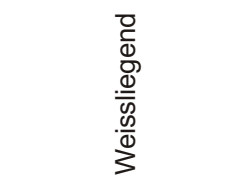 } \\
\hline
\end{tabular}




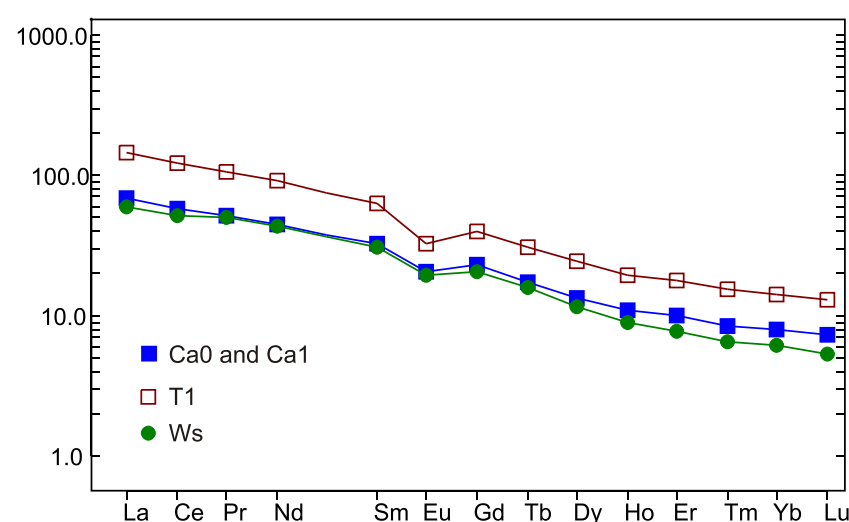

Fig. 3. Chondrite-normalized REE average concentrations for Basal Limestone and Zechstein Limestone carbonates ( $\mathrm{CaO}$ and Ca1), Kupferschiefer shales (T1) and Weissliegend sandstones (Ws)

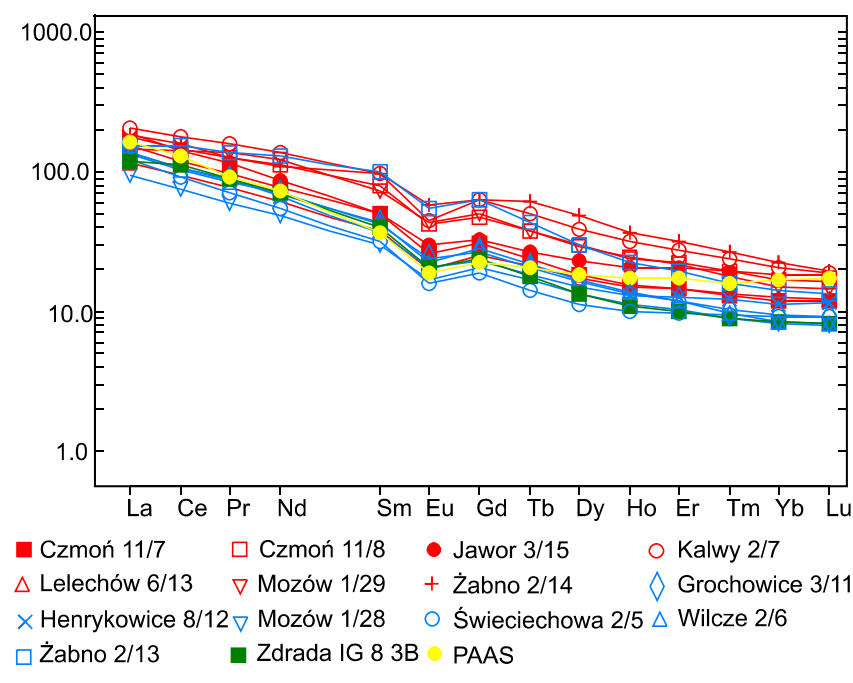

Fig. 4. REE diagram normalized to chondrite for the Kupferschiefer samples grouped into oxidized (red) and reduced (blue) rocks including the reduced sample from the Zdrada IG 8 borehole and the PAAS standard

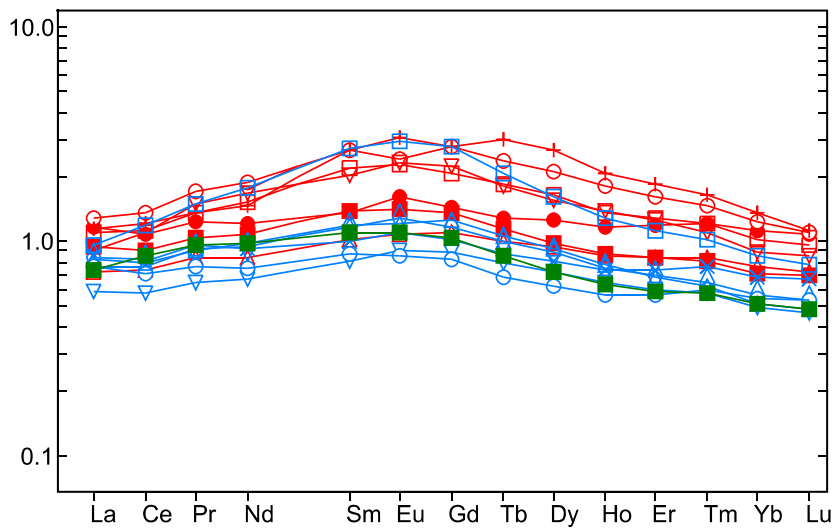

Fig. 5. REE diagram normalized to PAAS for the Kupferschiefer samples grouped into oxidized (red) and reduced (blue) rocks including the reduced sample from the Zdrada IG 8 borehole

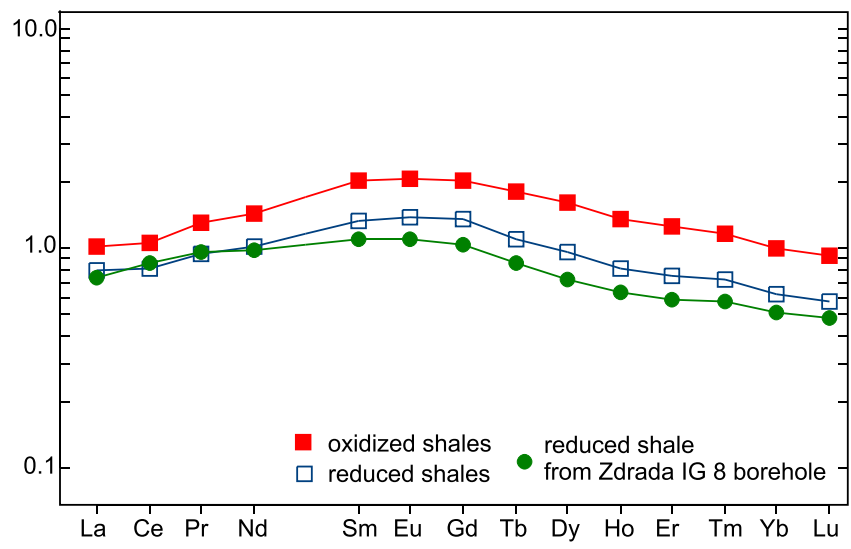

Fig. 6. PAAS-normalized REE average concentrations for the Kupferschiefer samples grouped into oxidized and reduced shales with respect to the reduced sample from the Zdrada IG 8 borehole

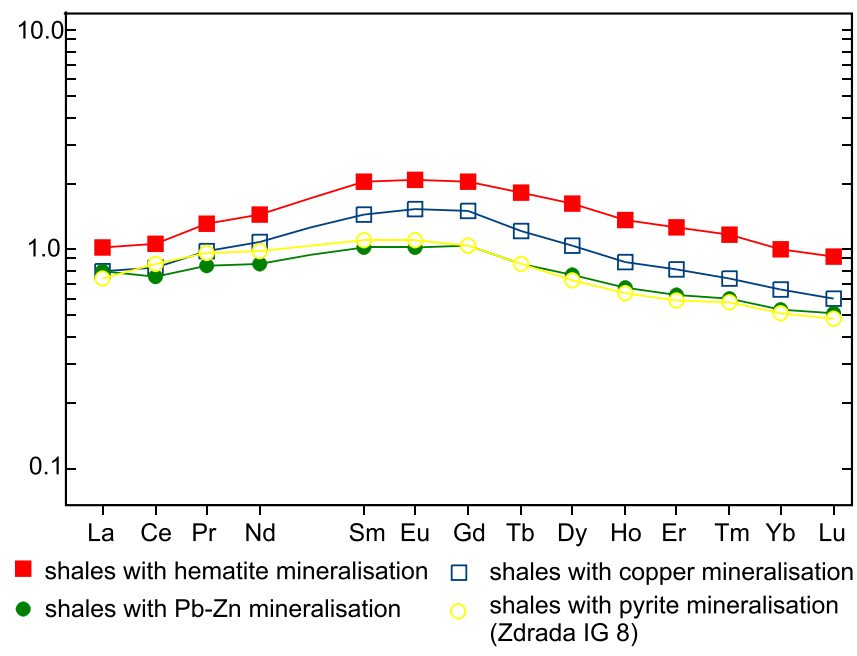

Fig. 7. PAAS-normalized REE average concentrations for the Kupferschiefer samples grouped into shales with predominant hematite, copper, and $\mathrm{Pb}-\mathrm{Zn}$ mineralisation with respect to the pyritic sample from the Zdrada IG 8 borehole

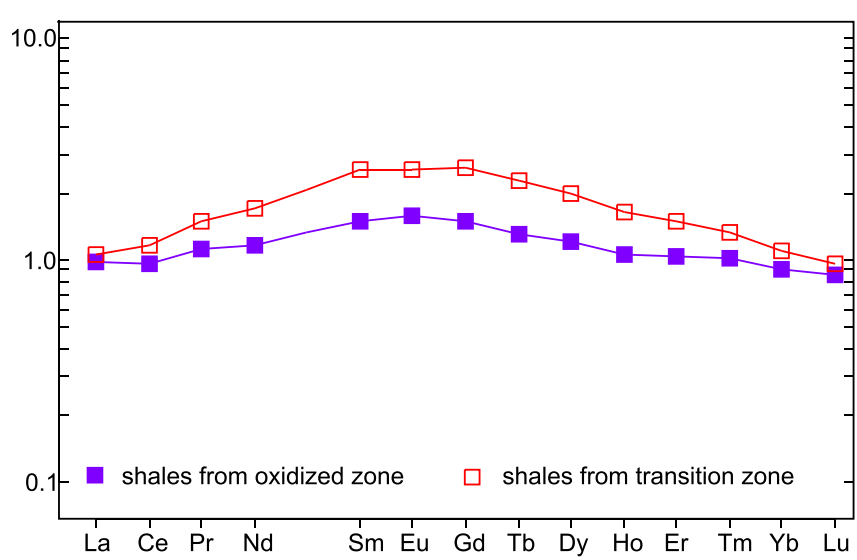

Fig. 8. PAAS-normalized REE average abundances for the Kupferschiefer samples grouped with respect to transition and hematite zones

For explanations see Figure 4 


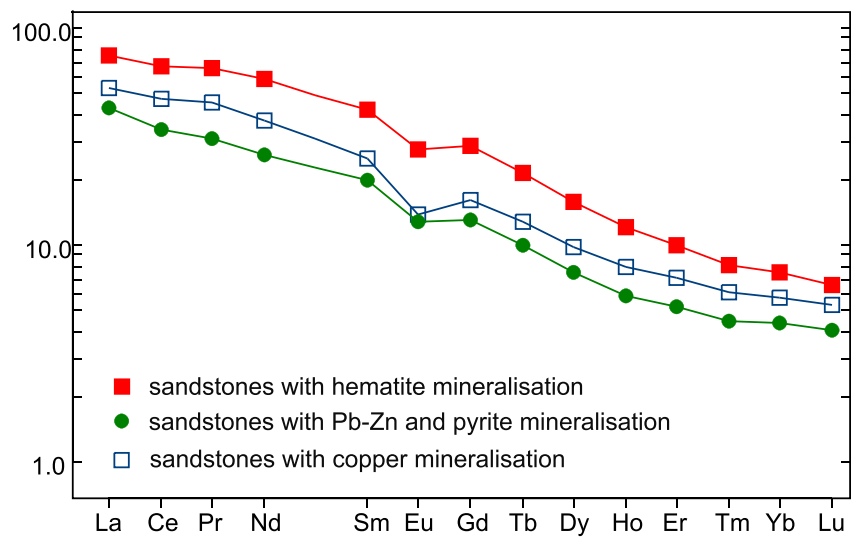

Fig. 9. Chondrite-normalized REE average abundances for the Weissliegend samples grouped into sandstones with hematite, copper, and $\mathrm{Pb}-\mathrm{Zn}$-pyrite mineralisation

ized signatures for the average REE content in carbonates, shales and sandstones (Fig. 3). The chondrite-normalized diagrams display a very similar shape of REE distribution curves, as well as the highest REE values for shales, enrichment in LREE, and negligible negative Eu anomaly for all types of lithological units.

\section{REE VARIATIONS IN OXIDIZED AND REDUCED ROCKS}

In the shales studied, $\Sigma$ REE varies in the range of 42-291 ppm, LREE range from 20 to $158 \mathrm{ppm}$, MREE from 13 to $95 \mathrm{ppm}$, and HREE are in the range of 3-25 ppm (Appendix 2). Based on REE analyses, average contents have been calcu- lated to show diversity with respect to oxidized vs. reduced shales (Table 2). The highest REE concentrations are recorded in the oxidized shales since $\Sigma$ REE is in the range from 145 to 291 ppm (with a mean of average $223 \mathrm{ppm}$ ), where LREE range between 86 and 158 ppm (average 125 ppm), and MREE (41-95 ppm, average $69 \mathrm{ppm}$ ) distinctly prevail over HREE values (10-25 ppm, average $16 \mathrm{ppm})$. The highest average values are exemplified by: Ce (85 ppm), Nd (47 ppm) and La (40 ppm). The average value of $Y$ (34 ppm) makes the sum of REY (REE with $\mathrm{Y})$ relatively high (257 ppm). A pronounced convex-up REE distribution pattern (Figs. 5 and 6 ) indicates that MREE (from $\mathrm{Nd}$ to $\mathrm{Gd}$ ) are enriched with respect to both LREE and HREE. This observation is strongly supported by several geochemical parameters (Table 3), such as a high average $\mathrm{Gd} / \mathrm{Gd}^{*}$ ratio (1.96), which clearly shows that oxidized rocks are strongly enriched in MREE. Furthermore, the high value of $\mathrm{Gd} / \mathrm{La}$ ratio (1.87) means that MREE are enriched compared to LREE. There is also a dominance of MREE over HREE ( $\mathrm{Gd} / \mathrm{Yb}=1.91 ; \mathrm{Nd} / \mathrm{Yb}=1.38)$ and $\mathrm{a}$ slight LREE prevalence over HREE (La/Yb = 1.03). However, the enrichments in MREE over the LREE are slightly greater than the enrichments of MREE relative to HREE.

Taking into account the division of shales into strongly oxidized hematitic rocks and slightly oxidized rocks typical of the transition zone, a significant enrichment in RREE (261 ppm) is recorded within the transition zone, including $\Sigma$ LREE (140 ppm), $\Sigma$ MREE (85 ppm) and $\Sigma$ HREE (20 ppm), Y (43 ppm) and Sc (16 ppm) in comparison to hematitic rocks (Appendix 2; Fig. 8). The values of coefficients, particularly specified by the $\mathrm{Gd} / \mathrm{Gd}^{*}$ (2.43), Gd/La (2.36), Gd/Yb (2.26), Nd/Yb (1.50) ratios, confirm that the transition zone in shales is typically characterized by strong MREE predominance over other groups (LREE, HREE).

Reduced shales in contrast to their oxidized counterparts are characterized by more variable $\Sigma$ REE values (from 115 to $253 \mathrm{ppm}$ ), but the average value is fairly low (163 ppm) (Table 2).

PAAS-normalized REE associated parameters in the Kupferschiefer and Weissliegend samples in relation to redox and mineralisation zones

\begin{tabular}{|c|c|c|c|c|c|c|c|c|c|c|c|}
\hline Lithostratigraphy & $\begin{array}{r}\text { Redox and mir } \\
\text { zon }\end{array}$ & eralisation & $\mathrm{Ce} / \mathrm{Ce}^{*}$ & $\mathrm{Eu} / \mathrm{Eu}^{*}$ & $\mathrm{Gd} / \mathrm{Gd}^{*}$ & $\mathrm{La}_{\mathrm{n}} / \mathrm{Yb}_{\mathrm{n}}$ & $\mathrm{Nd}_{\mathrm{n}} / \mathrm{Yb}_{\mathrm{n}}$ & $D y_{n} / Y b_{n}$ & $\mathrm{Gd}_{\mathrm{n}} / \mathrm{La}_{\mathrm{n}}$ & $\mathrm{Sm}_{\mathrm{n}} / \mathrm{Nd}_{\mathrm{n}}$ & $\mathrm{Gd}_{n} / \mathrm{Yb}_{\mathrm{n}}$ \\
\hline \multirow{8}{*}{ Kupferschiefer } & \multirow{2}{*}{ redox zones } & oxidized & 0.93 & 1.05 & 1.96 & 1.04 & 1.38 & 1.54 & 1.87 & 1.36 & 1.91 \\
\hline & & reduced & 0.94 & 1.05 & 1.83 & 1.36 & 1.67 & 1.49 & 1.57 & 1.22 & 2.08 \\
\hline & \multirow{4}{*}{$\begin{array}{c}\text { mineralisation } \\
\text { zones }\end{array}$} & hematite & 0.93 & 1.05 & 1.96 & 1.04 & 1.38 & 1.54 & 1.87 & 1.36 & 1.91 \\
\hline & & copper & 0.94 & 1.08 & 1.97 & 1.27 & 1.62 & 1.52 & 1.73 & 1.26 & 2.14 \\
\hline & & $\mathrm{Pb}-\mathrm{Zn}$ & 0.92 & 1.00 & 1.60 & 1.50 & 1.64 & 1.46 & 1.31 & 1.19 & 1.99 \\
\hline & & Pyrite & 1.01 & 1.02 & 1.71 & 1.44 & 1.92 & 1.42 & 1.42 & 1.11 & 2.05 \\
\hline & \multirow{2}{*}{ oxidized zone } & hematite & 0.92 & 1.07 & 1.61 & 1.10 & 1.30 & 1.34 & 1.51 & 1.27 & 1.66 \\
\hline & & transition & 0.95 & 1.03 & 2.43 & 0.96 & 1.50 & 1.80 & 2.36 & 1.47 & 2.26 \\
\hline \multirow{5}{*}{ Weissliegend } & \multirow{2}{*}{ redox zones } & oxidized & 0.91 & 1.25 & 2.84 & 1.06 & 1.78 & 1.90 & 2.67 & 1.44 & 2.82 \\
\hline & & reduced & 0.90 & 1.18 & 2.22 & 1.02 & 1.42 & 1.54 & 2.16 & 1.45 & 2.18 \\
\hline & \multirow{3}{*}{$\begin{array}{c}\text { mineralisation } \\
\text { zones }\end{array}$} & hematite & 0.91 & 1.25 & 2.84 & 1.06 & 1.78 & 1.90 & 2.67 & 1.44 & 2.82 \\
\hline & & copper & 0.91 & 1.10 & 2.18 & 1.00 & 1.48 & 1.54 & 2.13 & 1.38 & 2.11 \\
\hline & & $\mathrm{Pb}-\mathrm{Zn}$ & 0.90 & 1.23 & 2.25 & 1.03 & 1.38 & 1.54 & 2.19 & 1.49 & 2.18 \\
\hline
\end{tabular}


Similarly, the values of $\Sigma$ LREE (68-131 ppm, average $96 \mathrm{ppm}$ ), ¿MREE (32-91 ppm, average $49 \mathrm{ppm}$ ) and $\Sigma$ HREE (7-15 ppm, average $9 \mathrm{ppm}$ ) in reduced shales are significantly lower than in oxidized shales. Due to the lower content of yttrium in reduced rocks, $\Sigma$ REY is also lower (186 ppm) than in the oxidized shales (257 ppm). PAAS-normalized REE signatures show ratios $(\mathrm{La} / \mathrm{Yb}=1.36, \mathrm{Gd} / \mathrm{Yb}=2.08, \mathrm{Nd} / \mathrm{Yb}=1.67)$ indicating stronger enrichments in LREE and MREE over HREE but weaker enrichment in MREE over LREE relative to oxidized rocks (Table 3 ).

As shown in Figure 4, chondrite-normalized oxidized shales are enriched in REE relative to reduced shales mineralized with sulphides. When normalized to PAAS, the values in oxidized shales generally exceed 1.0 indicating that REE are more abundant in these lithologies than in PAAS, whereas the normalized REE abundances are usually less than 1.0 in reduced shales revealing depletion relative to PAAS (Figs. 5-7). Characteristically, despite the similarity in the overall distribution of REE, oxidized shales are characterized by a distinct enrichment in both REE and MREE not only in comparison with reduced shales, but also in relation to the PAAS standard. MREE enrichments are particularly noticeable in PAAS-normalized REE distribution diagrams, where apparent convexities are occupied by MREE.

In the case of sandstones, similar trends of REE variation to those recorded in shales are observed (Appendix 2; Table 2). Oxidized sandstones have higher REE contents than reduced ones (114 and 65 ppm respectively), including: LREE (59 and $36 \mathrm{ppm}$ ), MREE (40 and $21 \mathrm{ppm}$ ) and HREE ( 8 and $4 \mathrm{ppm}$ ). Similar are $Y$ and Sc abundances. The dominance of MREE over other groups is supported by some ratios (Table 3 ): $\mathrm{Gd} / \mathrm{Gd}^{*}$ (2.84 and 2.22 values for oxidized vs. reduced sandstones, respectively), $\mathrm{Gd} / \mathrm{La}$ (2.67 and 2.16, respectively) and $\mathrm{Gd} / \mathrm{Yb}(2.82$ and 2.18, respectively). Among MREE, particularly high concentrations of $\mathrm{Gd}$ are typical for oxidized sandstones (Fig. 9), resulting in the significant positive anomaly $(\mathrm{Gd} / \mathrm{Yb}=2.82)$, even higher than in the case of oxidized shales $(\mathrm{Gd} / \mathrm{Yb}=1.91)$.

\section{REE VARIATIONS IN MINERALISATION ZONES}

Shales with different types of mineralisation are characterized by various REE contents and specific distribution patterns (Table 2 and Fig. 7). There is a consistent decreasing trend in ¿REE from hematite-containing shales (223 ppm) through shales with copper mineralisation (172 ppm) to shales with $\mathrm{Pb}-\mathrm{Zn}(147 \mathrm{ppm})$ and pyrite mineralisation in the Zdrada IG 8 borehole (158 ppm). LREE exhibit a similar tendency (with mean of 125, 99, 90 and 96 ppm, respectively in oxidized, copper, $\mathrm{Pb}-\mathrm{Zn}$ and pyrite shales), as well as MREE $(69,53,41$ and $45 \mathrm{ppm}), \operatorname{HREE}(16,10,8$ and $8 \mathrm{ppm}), \mathrm{Y}(35,24,18$ and 17 $\mathrm{ppm})$ and $\mathrm{Sc}(15,9,8$ and $9 \mathrm{ppm})$. The flattest diagram is typical of shales mineralized predominantly by pyrite (Zdrada IG 8 borehole) and shales with $\mathrm{Pb}-\mathrm{Zn}$ mineralisation (Święciechowa 2 and Wilcze 2 boreholes), but the most convex-up are REE distribution patterns for shales with copper and hematite mineralisation. Hence, a significant decrease in MREE is observed going from oxidized to pyritic shales $\left(\mathrm{Gd} / \mathrm{Gd}^{*}\right.$ ranging from 1.96 in oxidized shale to 1.71 in pyritic shales as well as $\mathrm{Gd} / \mathrm{La}$ from 1.87 to 1.42 respectively). A similar trend is also typical in the case of sandstones (Table 3 and Fig. 9).

It is obvious that oxidized rocks (including weakly oxidized rocks from the transition zone) are found to have the highest MREE concentrations, which is illustrated by characteristic convexities in PAAS-normalized REE curves compared with less convex patterns for samples with copper and $\mathrm{Pb}-\mathrm{Zn}$ mineralisa- tion, and with the most flat REE diagram pattern typical for barren rocks with pyrite mineralisation (Fig. 7). All samples show a very weak negative $\mathrm{Ce} / \mathrm{Ce}^{*}$ anomaly and nearly all (except two samples) a slightly positive Eu/Eu* anomaly (Table 3 ), regardless of the mineralisation zone.

The slightest differences in the REE patterns in the shale specimens with regard to different mineralisation zones are observed in the case of LREE, despite a similar rising trend from the pyrite to hematite mineralisation zone (Fig. 7), whereas a significant increasing trend of HREE dominance over LREE is finely marked ( $\mathrm{La} / \mathrm{Yb}$ decreases from 1.04 in hematitic shales to $1.4-1.5$ in $\mathrm{Pb}-\mathrm{Zn}$ and pyritic shales).

In turn, a fairly visible decline in the value of $L a / Y b$ and $\mathrm{Nd} / \mathrm{Yb}$ ratios from pyritic to hematitic shales indicates an increasing enrichment in HREE within shales (with copper and hematite mineralisation) with respect to light REE, such as La and $\mathrm{Nd}$. The outcome of this trend is flat REE distribution diagrams for shales with hematite mineralisation in the direction towards HREE compared to shales with copper mineralisation, which are characterized by more convex curves (Fig. 7). In the case of sandstones there is a clear ramp-like steep peak in the direction towards the lightest REE (Table 3), which means an impoverishment of copper-bearing and hematite sandstones in HREE compared with sandstones containing $\mathrm{Pb}-\mathrm{Zn}$ and pyritic mineralisation.

\section{INTERPRETATION AND DISCUSSION}

In the marine sedimentation dominated by deposition of clay and organic matter, the distribution of REE depends primarily on the composition of terrigenous source material, biogenic material composition, and redox conditions in bottom waters (e.g., Piper, 1974; McLennan, 1989; German et al., 1991; Jiang et al., 2006; Zanin et al., 2010). Modern sediments deposited under reducing conditions are characterized mostly by relatively flat REE patterns, with a slight HREE enrichment (de Baar et al., 1988; Piper and Bau, 2013). However, the ultimate REE distribution in sediments is commonly governed by diagenetic processes (Schieber, 1988; Milodowski and Zalasiewicz, 1991; Shields and Stille, 2001; Bouch et al., 2002) or interaction of sediments with migrating fluids, which tends to increase REE contents and concentrate preferentially the middle REE (de Baar et al., 1988; Gieré, 1996; Zwingmann et al., 1999; Wood, 2003). Because of this, REE distribution diagrams for various kinds of black shales can exhibit high variability where metalliferous black shales are usually more strongly enriched in REE than typical black shales (Table 1; Huyck, 1989; Jiang et al., 2006).

The primary source of REE and the origin of their enrichments in rocks of the Kupferschiefer series are not well understood. These issues have been broadly discussed by Mayer et al. (1992), Bechtel et al. (2001b), Michalik (2001) and Sawłowicz (2013), but significant differences between total REE and MREE concentrations in the transition zone in comparison with samples containing hematite and copper mineralisation lean toward supplementing an earlier interpretation. According to Mayer et al. (1992), differences in concentrations of REE may reflect various compositions of source material and modification by diagenetic processes rather than changes during short-distance transport of terrigenous material to the sedimentary basin. Bechtel et al. (2001b) maintained that REE, just like copper, gold, platinum and many other metals, were derived from either the underlying Rotliegend sandstones and volcanics, the 
Variscan basement rocks, or the Kupferschiefer shales whose metals were mobilized by saline, oxidizing fluids released during Triassic intra-continental rifting. Zwingmann et al. (1999) suggested that REE might have been supplied to migrating fluids through feldspar alteration, and Michalik (2001) pointed out to the large potential role of iron oxide coatings, feldspars and monazite, present in the Rotliegend rocks, for the release of REE. It is believed that the differences in REE contents cannot be generally provenance-dependent, but they were rather caused by post-sedimentary processes related to their desorption from large volumes of ascending solutions (Sawłowicz, 2013).

Although the primary sources of REE are enigmatic, the general similarity shown in the chondrite or PAAS-normalized REE patterns for different lithological samples that come from different localities indicates that REE may have originated from a similar terrigenous source. It may be argued that rare earth elements, as copper, silver, lead, zinc, gold and platinum, were derived from the pre-Permian basement and (or) from the Rotliegend siliciclastic and volcanic rocks. Weathering of bedrock and erosional processes led to deposition of vast masses of detrital material accumulated in the Rotliegend basin in the form of red-beds. REE are assumed to be sourced from dissolution of various rock-forming minerals (feldspar, apatite, fluorocarbonate, titanite, zircon, monazite), volcanic detritus, disseminated hematite, clay-hematite coatings on detrital grains, and mudstone interbeds under acidic conditions, as suggested for other geological settings (e.g., Piper, 1974; McLennan, 1989; Milodowski and Zalasiewicz, 1991; Wood, 2003). Michalik (2001) challenged the assumption that REE were derived from decomposition of feldspars or diagenetic transformation of monazite in oxidizing conditions. As a result of chemical weathering and interaction of the formation fluids with Rotliegend rocks, REE might have been preferentially scavenged by clay/Fe-oxyhydroxide coatings on detrital components of the red beds (Hartmann et al., 1997; Zwingmann et al., 1999). This process has also been observed in many geological settings (e.g., Bau, 1999; Protano and Riccobono, 2002; Verplanck et al., 2004; Welch et al., 2009; Ma et al., 2011).

The ability of aqueous solutions to mobilize REE from the host rocks depends mainly on the stability and availability of REE ligands (Migdisov et al., 2016). Generally, REE exhibit a significant tendency to form $\mathrm{Cl}^{-}, \mathrm{F}^{-}$and $\mathrm{SO}_{4}^{2-}$ complexes, but in warm and mildly acidic saline brines they commonly form stable complexes with chloride which can transport appreciable amounts of REE at low pH (Gosselin et al., 1992; Gammons et al., 1996; Gieré, 1996; Brugger et al., 2006; Mayanovic et al., 2007; Migdisov et al., 2016).

As commonly argued, Rotliegend formation waters were characterized by a relatively low $\mathrm{pH}$, high $\mathrm{Eh}$ (in the stability field of hematite), considerable salinity, high concentration of chloride ions, and elevated temperatures increasing the ability of the fluid to transport metals (e.g., Jowett et al., 1987; Wodzicki and Piestrzyński, 1994; Oszczepalski, 1999; Speczik et al., 2003). Such fluids can dissolve as little as up to 4 ppb REE (Gieré, 1996; Jaireth et al., 2014), but chloride complexation and REE mobility sufficiently increases with increasing temperature (Gammons et al., 1996; Gieré, 1996; Migdisov et al., 2016). Although weaker than other aqueous complexes of REE, chloride species, like REECl ${ }^{2+}$, can play a significant role in REE transport due to the considerable concentrations of chloride in natural solutions (Migdisov et al., 2016). As a result of water-rock interactions involving fluids with a high concentration of chloride ligands, REE are thought to have been liberated as REE-chloride complexes by groundwater and transported to the Lower Zechstein rocks by mineralizing solutions. It should be noted, however, that even though chloride transport of REE is plausible and widely accepted, low solubility of REE in the form of chloro-complexes shows that their delivery to the lowermost Zechstein to form enrichments was probably not decisive because stability of REE chloride species at low temperature is not high (Migdisov et al., 2016). Although many hydrothermal REE deposits are assumed to form from fluoride complexes, extremely low solubility of REE-fluorides in low-temperature solutions may indicate that possible contribution of fluoride species to REE transport is much less significant (Migdisov et al., 2016). The other obvious candidates for transporting REE are sulphates (Sholkovitz, 1995; Gieré, 1996; Johannesson et al., 1996; Wood, 2003; Migdisov et al., 2016).

A highly significant positive relationship between the REE enrichments and oxidative alteration is obvious; nevertheless the formation of elevated REE concentrations in oxidized rocks seems unclear. On the basis of relics of sulphide minerals surrounded by hematite pigment and copper sulphide replacements by hematite and other iron oxides in the oxidized zone, it should be assumed that alteration of sulphides and organic matter generated acidic fluids with appreciable concentrations of sulphate from oxidation of sulphides. This oxidation resulted in the precipitation of iron oxides. The acidification following the oxidation enhanced the mobilization and leaching of various elements from altered rocks. As a result, the oxidized rocks are significantly depleted in most of the base metals (Appendix 1). At a weakly acidic $\mathrm{pH}$, mobility of REE may significantly increase due to formation of the stable sulphate ligand, mainly $\mathrm{REESO}_{4}^{+}$and $\mathrm{REE}\left(\mathrm{SO}_{4}\right)^{2-}$, enhancing the release of REE from rocks (Sholkovitz, 1995; Gieré, 1996; Wood, 2003; Zhao et al., 2007; Migdisov et al., 2016). Additionally, the acidification may have promoted dissolution of carbonates and hydrolysis of clay minerals at acidic $\mathrm{pH}$, liberating additional amounts of REE (Olias et al., 2005). For these reasons, it is believed that low-pH solutions dominated by sulphate ligands, such as those generated by oxidation processes, enhanced leaching of REE from the altered rocks.

It is suggested that REE deposition in the hematitic zone was caused by interaction of oxidizing solutions with rocks, resulting in destabilization of sulphate complexes that can occur due to changing $\mathrm{pH}$, Eh and temperature conditions of migrating solutions (cf. Gieré, 1996; Gammons et al., 1996). Many researchers have argued that REE sulphate complexes in acidic environments can be adsorbed onto the Fe-Al-Mn hydrous oxides (Johannesson et al., 1996; Bau, 1999; Protano and Riccobono, 2002; Gammons et al., 2003; Verplanck et al., 2004; Brugger et al., 2006; Zhao et al., 2007; Welch et al., 2009). Because the flow of mineralizing solutions took place within the carbonate-rich rocks of the Kupferschiefer series, subsequent fluid-rock exchange reactions (including dissolution of carbonates) neutralized acidity of the solution (cf. Worrall and Pearson, 2001; Verplanck et al., 2004; Olias et al., 2005; Migdisov et al., 2016). After the release of REE into solution, as acidic fluids were buffered to a higher $\mathrm{pH}$ by carbonates, reactive Fe-oxyhydroxides scavenged REE due to destabilization of REE sulphate ligands, removing them from oxidizing fluids. Consequently, it seems likely that the formation of REE enrichments (including MREE) in the oxidized rocks was favoured by interaction of the REE-enriched fluid with wall rocks containing efficient neutralizers in the form of carbonate, and followed by their coeval uptake by precipitating ferric oxyhydroxides. Sorption, not only by hematite but also by clay, silica and alumina, can be also responsible for REE concentration (cf. Protano and Riccobono, 2002; Gammons et al., 2003; Piasecki and Sverjensky, 2008; Zanin et al., 2010; Ma et al., 2011), therefore uptake by clay minerals (Sawłowicz, 2013), particularly by neomorphic illite (Bechtel et al., 1999, 2001b) and by carbon- 
ates (Michalik, 2001), can be also considered. Variability of REE concentrations, with respect to different lithologies are assumed to be associated with the content of clay material (Mayer et al., 1992). As demonstrated by European Shale/REE normalized curves, REE contents of the silicate fraction show signatures similar to the whole rock analyses, indicating that silicates are the main carriers of REE (Bechtel et al., 2001b). Glauconite locally dispersed in oxidized rocks can be an additional site for concentration of some REE (cf. Stille and Clauer, 1994). Likewise, iron sulphides and organic matter are suggested as possible sites for REE adsorption (cf. Welch et al., 2009). Michalik (2001) demonstrated an increase of REE content in the uppermost part of the sandstones that may have been caused by the formation of authigenic REE carbonate and phosphate minerals in carbonate cement of the topmost Weissliegend or due to the presence of detrital heavy minerals and feldspar.

It should be noted that slightly oxidized shales, typical for the transition zone, are more enriched in REE than intensely oxidized hematitic shales from centres of the Rote Fäule areas (Fig. 8). It is postulated that REE were leached from the most oxidized rocks, redistributed along the pathways of fluids, and formed enrichments in the transition zone. By this stage, the conversion of intermediate REE-bearing Fe-oxyhydroxide phases to hematite, and the formation of authigenic illite and (or) the recrystallisation of illite (Bechtel et al., 1999) could promote subsequent mobilization of REE and their transport from mostly oxidized centres of alteration, and later reprecipitation of REE in localities close to the expanded redox front.

The origin of MREE enrichments in the Kupferschiefer is unclear, as they may have been inherited from the source rock or mineralizing fluids; alternatively it can be a consequence of solid-liquid exchange reactions. Although the PAAS-normalized REE curves have similar shapes (Figs. 5-8), it is evident that oxidized and copper-bearing rocks are characterized by more pronounced enrichment of MREE over LREE and HREE than $\mathrm{Pb}, \mathrm{Zn}$, and pyrite-mineralized rocks.

Several mineral phases or a combination of iron oxides, phosphates, carbonates, organics and sulphides have been suggested as MREE sources (Johannesson et al., 1996). Although organic matter and sulphides are not effective concentrators of REE, they may accumulate more $(\mathrm{M}+\mathrm{H})$ REE than LREE (Jiang et al., 2006; Zanin et al., 2010). Locally encountered rocks of the Kupferschiefer series enriched in $\mathrm{P}_{2} \mathrm{O}_{5}$ or containing phosphate minerals (e.g., Kucha, 1982; Mayer and Piestrzyński, 1986; Hartmann et al., 1997; Michalik, 2001; Sawłowicz, 2013) suggest the possibility of REE liberation from phosphates which are commonly enriched in MREE (e.g., Hannigan and Sholkovitz, 2001; Shields and Stille, 2001; Bouch et al., 2002).

A number of possible processes have been suggested to explain the enrichment of MREE in natural waters, including the original composition of source rocks, solid-liquid exchange reactions, dissolution of MREE-rich mineral phases, leaching of MREE-enriched mineral surface coatings, fractionation by surface/solution reactions between MREE-enriched minerals and acid waters, sulphate and (or) phosphate complexation, and the combined action of different mechanisms (Gosselin et al., 1992; Sholkovitz, 1995; Gieré, 1996; Gammons et al., 2003). Strong consideration is given to chemical properties of mineralizing solutions (low $\mathrm{pH}$, high salinity, elevated temperature and the presence of ligands) that facilitate fluid-rock interactions (Johannesson et al., 1996; Worrall and Pearson, 2001; Protano and Riccobono, 2002; Verplanck et al., 2004), however, desorption processes (Gosselin et al., 1992; Protano and Riccobono,
2002; Ma et al., 2011) and leaching of certain mineral phases, such as hematite, phosphates, clay minerals, carbonates or organic matter have been proposed as the most probable processes that may be responsible for MREE enrichments in fluids (Sholkovitz, 1995; Johannesson et al., 1996; Cruse et al., 2000, Hannigan and Sholkovitz, 2001; Shields and Stille 2001; Welch et al., 2009; Zanin et al., 2010).

There is plenty of evidence that low-pH solutions rich in sulphates are more capable of being enriched in MREE through sulphate complexation because MREE sulphate ligands are most stable compared with LREE and HREE equivalents, indicating greater MREE mobility (Johannesson et al., 1996; Gammons et al., 2003; Zhao et al., 2007). It was clearly demonstrated (e.g., Bau, 1999; Worrall and Pearson, 2001; Protano and Riccobono, 2002; Verplanck et al., 2004; Olias et al., 2005; Welch et al., 2009; Ma et al., 2011) that metal sulphide-containing rocks affected by acidic washing tend to be depleted in MREE whereas fluids resulting from oxidative alteration present MREE-enriched signatures and that ferric oxyhydroxides played a crucial role in both the REE uptake and removal. As previously suggested, deposition of MREE, like REE, occurred due to destabilization of REE-sulphate complexes through $\mathrm{pH}$ neutralization by carbonates, or through processes that reduce the sulphur, and transform it into species, such as thiosulphates, sulphides, or native sulphur (Migdisov et al., 2016). This may also indicate that a short-distance transport of REE might have been facilitated by processes that transform sulphate into metastable intermediate sulphur oxyanions during fluid percolation through the redox transition (Oszczepalski, 1994, 1999; Piestrzyński and Wodzicki, 2000). A distinct relationship between distribution of REE, oxidative alteration and metal zonation strongly supports the suggestion of Bechtel et al. (2001b) that mid-REE enrichment is probably related to the formation of insoluble mineral phases associated with the silicate fraction accompanied by iron oxyhydroxides, as evidenced by similar REE distributions for both rock samples and silicate fraction. They conclude that MREE have been preferentially partitioned into secondary ferric iron precipitates and authigenic illite, which were formed during oxidation. Therefore, it seems that the most likely cause of elevated MREE patterns can be attributed to dissolution of MREE-enriched Fe-oxyhydroxides and, to a lesser extent, phosphates. Subsequently, MREE were presumably scavenged from migrating fluids by aged iron-oxyhydroxides and coeval authigenic illite. Since the highest MREE concentrations typically occur in external parts of the oxidized rocks studied (Fig. 8), one can assume that these enrichments are the consequence of acidic sulphate solution flow outward from the core of the ore system exhibiting the most extensive alteration, while fluids expanded the hematitic alteration front (Fig. 10).

Slightly different signatures for shales from the Żabno 2/13 and, to a lesser extent, from the Kalwy 2/7 and Czmoń 11/8 boreholes (Figs. 4 and 5) that are situated at the northern side of the Wolsztyn palaeo-high, as compared to other signatures, may be attributed to a number of factors including mixing from many sources and intensity of remobilization.

\section{A RESOURCE PERSPECTIVE}

The term "rare earths" creates confusion, because the average content of REE in the crust (approx. 148 ppm; Table 1) is higher than most of industrial base metals, such as copper, zinc, lead, molybdenum, nickel, tin or tungsten, and the concentration of the least abundant lutetium is many times higher than of gold. 


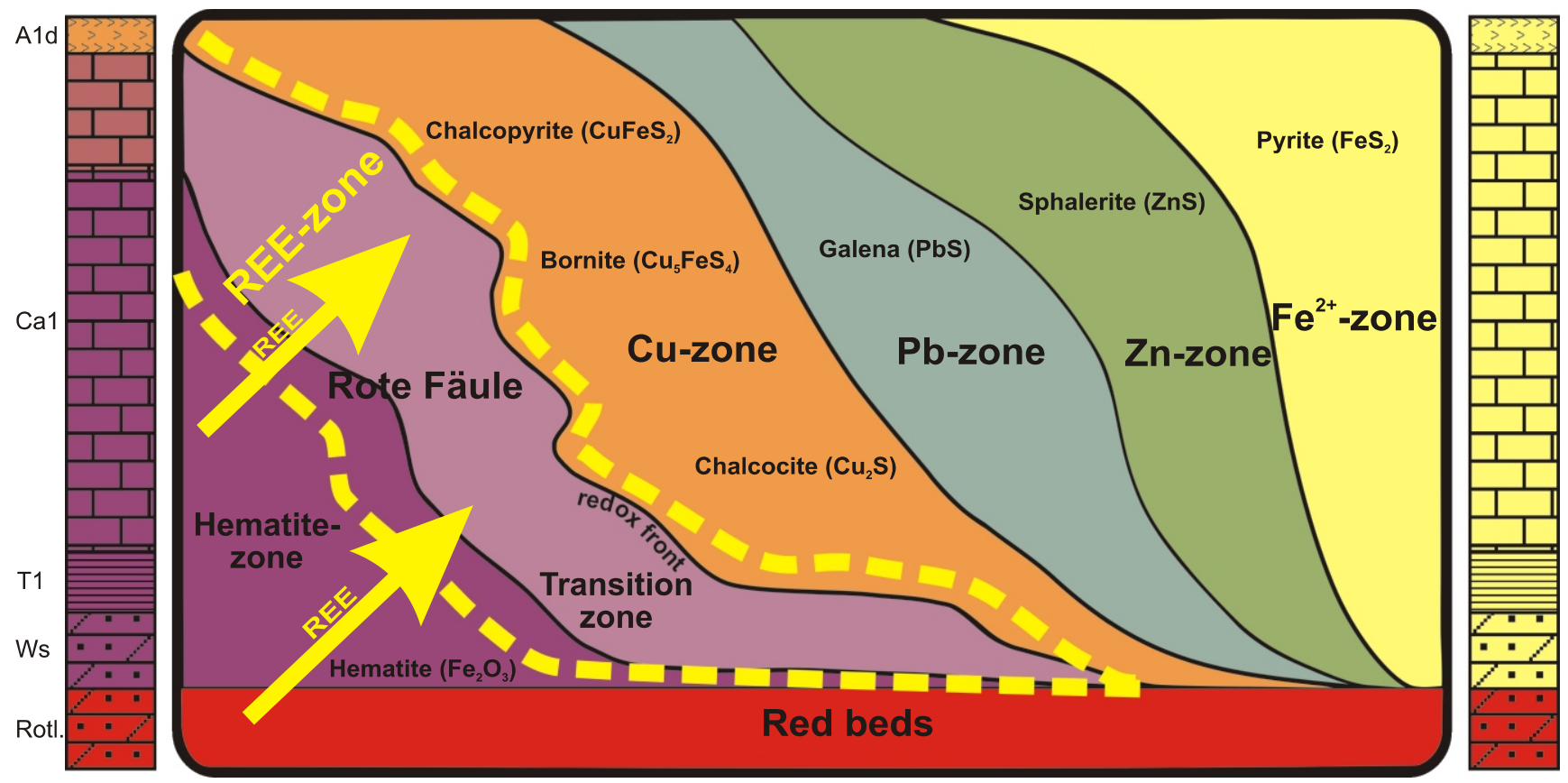

Fig. 10. Schematic section of the Kupferschiefer series showing the REE-enriched zone in relation to the oxidized Rote Fäule and the metal zoning with respect to the enclosing strata

However, in contrast to these metals, REE exhibit a poor tendency to form ore concentration. Most of the resources are located in China, Brazil, USA, India, Australia, Malaysia, Canada and South Africa. In Europe, insignificant deposits, resources and occurrences are found in Sweden, Norway, Finland, Spain, Turkey and Germany (Simandl, 2014; Goodenough et al., 2016). The most important localities classified as REE resource are associated with carbonatites, syenites, pegmatites, peralkaline igneous rocks, monazite-apatite veins, clays, laterite, bauxite, heavy minerals deposits, uranium-bearing conglomerates, xenotime sandstones, phosphorites, skarns, greisens, iron oxide copper-gold (IOCG) ores, and deep-sea manganese nodules and muds. Global mining of REE (with Y) is currently approx. 120 thousand tons (2013) and ca. 95\% of production comes from China and only $5 \%$ come from other producers including USA, India, Russia, Australia, Vietnam, Brazil and Malaysia (Long et al., 2012; Simandl, 2014).

Global REE resources are higher than expected consumption in the 21st century. However, concentration of production in China, the supply risk, abrupt variations in prices, and recent REE shortages for the production of high-tech applications have forced companies to invest in exploration. The measure of this activity is almost 600 active exploration projects, of which approx. 400 perform drilling exploration and prospecting (Simandl, 2014). World REE-demand growth is estimated to be increasing at a rate of about $8 \%$ per year due to technological progress (Long et al., 2012). As a result of market shortages, non-delivery risk is likely, hence the EU recognized REE as critical for its economic development (Galos et al., 2012). This applies particularly to MREE-HREE, for which there is the highest demand, reflected by the highest prices exceeding $\$ 100 / \mathrm{kg}$ of metal (Dy, Nd and Pr), or even $\$ 1,000 / k g$ (Tb and Eu).

As is apparent from the review of different types of documented REE deposits, the lowest average REO (rare-earth oxides) grade ranges within 100-1000 ppm (Long et al., 2012; Jaireth et al., 2014; Goodenough et al., 2016), but the average REO content in most deposits with proven reserves typically ex- ceeds the value of $1 \%$. Insights from comparison of Table 2 and Appendix 2 show that the maximum concentration of REE in the copper shale ( $\Sigma R E E$ in the range of $140-230 \mathrm{ppm}$ ) only marginally exceeds the average content in the Earth's crust (140-150 ppm) and world shales (150-390 ppm; Table 1). The reported average content of REE in the transition zone (261 ppm) and, to a lesser extent, in hematitic and copper-rich shales (194 and 172 ppm, respectively) fall within the range between black shales and metalliferous black shales (REE average 185 and 390 ppm, respectively; cf. Table 1), which means that even the richest Kupferschiefer samples ( $\Sigma$ REE 291 ppm in oxidized shales and up to $253 \mathrm{ppm}$ in shales with copper mineralisation) do not contain substantial concentration of REE. In the case of sandstone and carbonate samples, abundances of REE are even smaller than in shales, but the REE content is also the highest in oxidized sandstone samples (average content is $114 \mathrm{ppm}$ at a maximum up to $186 \mathrm{ppm}$ ). Therefore, the Kupferschiefer series, due to relatively low REE concentrations compared to documented REE deposits, does not seem to be a prospective source, barring the appearance of economic need, particularly in parts of the oxidized areas, in which REE coexist spatially with gold and PGE. The REE deposits associated with black shale are extremely rare (Gieré, 1996; Long et al., 2012; Jaireth et al., 2014; Simandl, 2014), excepting a few polymetallic black shales (Weng et al., 2015), phosphorite-bearing shales (e.g., Cruse et al., 2000), black slates (Loukola-Ruskeeniemi and Lahtinen, 2013) or shales with IOCG hydrothermal mineralisation (Simandl, 2014). REE in several other shale-related polymetallic deposits occur as accompanying commodities (e.g., Jiang et al., 2006; Zanin et al., 2010), however, there is little information about economic utility.

Modes of occurrence of REE in the examined samples are not well understood. Similar REE patterns for sandstones, shales and carbonates may suggest that REE are mainly linked with terrigenous material, iron oxides and, to lesser extent, with phosphates, carbonates, accessory minerals as well as with kerogen and sulphides (in reduced rocks) or glauconite (in 
slightly oxidized rocks). The likely presence of rare earths within the structure of various REE-bearing mineral phases, that are difficult to process, makes it difficult to consider the mode of extraction, recoverability, and related costs (Long et al., 2012; Weng et al., 2015).

The considering of the Kupferschiefer series as a prospective source of REE (even in the case of efficient recovery technology) is not currently justified from an economic point of view. However, since almost all investigated rock samples (especially oxidized shales) are characterized by elevated REE contents, it is not unlikely to find even higher (than disclosed today) concentrations that may occur locally in undiscovered oxidized localities. Regional prospectivity assessment of REE could be particularly useful in areas with Rote-Fäule-related Au-Pt-Pd mineralisation, when considering the possibility of their combined production.

\section{CONCLUSIONS}

This study of REE concentrations in 27 samples of the Kupferschiefer series from 10 boreholes revealed large-scale regularity in variability of their concentration in terms of areas with different geochemical zones. Distinct differences in REE concentrations (including LREE, MREE and HREE) in shales are observed on the REE diagrams normalized both to chondrite and to PAAS with respect to redox changes as well as with regard to type of mineralisation. The highest $\Sigma$ REE and $\Sigma$ MREE concentrations ( 261 and 85 ppm on average, respectively) are found to be associated with oxidized shales of the transition zone, which is reflected in relatively symmetric upward convex REE distribution patterns in relation to PAAS, whereas slightly flatter REE profiles characterize copper shales, and most of $\mathrm{Pb}-\mathrm{Zn}$ and pyritic shales. Concentrations of REE versus the transition zone tend to be lower in the hematite zone occupying centres of the Rote Fäule areas (223 ppm) and in successive zones with copper (194 ppm), Pb-Zn (147 ppm) and pyrite (158 ppm) zones. Very similar trends can be also observed with respect to $Y$ and Sc. The abundances of REE in sandstones and carbonates are lower than in shales, but the trend of their increasing concentration towards oxidized lithologies is also noted.

The results are consistent with a recent genetic model for the Kupferschiefer mineralisation, arguing for the migration of metalliferous brines outward from the cores of the Rote Fäule areas into the reduced sediments. The regional association between Rote Fäule alteration, metal zonation, and distribution of REE enrichments indicates that both REE and base metals were introduced by the same hydrothermal fluids during progressive evolution of the mineralizing system. The striking feature is that the upgraded REE abundances in oxidized rocks coincide with the Rote Fäule-related gold and PGE concentrations, suggesting that both types of enrichment were likely a result of similar fluid/rock interactions.

The highest concentration of REE, especially MREE, within the Rote Fäule, testifies to remobilization and movement of rare earth elements by relatively low-pH and sulphate-dominated fluids MREE>HREE>LREE, and their deposition superimposed on the original REE distribution along flow paths of mineralizing solutions. This is indicated by a systematic decrease in REE (especially MREE) from oxidized rocks, through rocks mineralized with copper sulphides up to the outermost $\mathrm{Pb}-\mathrm{Zn}$ and pyrite zones. The lowest average concentrations of REE (including LREE, MREE and HREE) were found in barren pyritic shales, since areas of the pyrite zone were not being subjected to extensive mineralizing processes.

A pronounced convex-up PAAS-normalized REE pattern indicating the highest enrichment in MREE within shales from the transition zone can be explained by leaching of altered rocks and lateral REE redistribution during migration of mineralizing fluids enriched in sulphate ligands, and followed by progressive fixation and concentration of REE (chiefly MREE) by secondary ferric iron precipitates and authigenic illite, which have been formed contemporaneously with oxidation.

Despite forecasted global REE-demand growth by new technologies, due to relatively low concentrations (compared to the average grade in REE deposits and occurrences), the dominance of LREE and MREE over the most economically valuable HREE, and the occurrence of REE within the matrix of rock-forming minerals, the Kupferschiefer series do not seem to be a valuable source of REE to economic levels. Nevertheless, the overall potential looks prospective regardless of the size or grade, particularly in relation to those parts of oxidized areas, where REE coexist spatially with Au and PGE.

Acknowledgements. This research was made possible through grant No. 61.6705.1301.00.0. from the Polish Geological Institute - National Research Institute to S.Z. Mikulski. I. Iwasińska-Budzyk, D. Karmasz and I. Wysocka (PGI-NRI) are acknowledged for chemical analysis. We thank PGNiG S.A. for admission to the borehole material. Z. Sawłowicz, S. Sutton and an anonymous reviewer are thanked for their constructive comments. T.M. Peryt is thanked for his comments on the manuscript and editorial guidance.

\section{REFERENCES}

Bau, M., 1999. Scavenging of dissolved yttrium and rare earths by precipitating iron oxyhydroxide: experimental evidence for $\mathrm{Ce}$ oxidation, Y-Ho fractionation, and lanthanide tetrad effect. Geochimica et Cosmochimica Acta, 63: 67-77.

Bechtel, A., Elliott, W.C., Wampler, J.M., Oszczepalski, S., 1999. Clay mineralogy, crystalinity and K-Ar ages of illites within the Polish Zechstein basin: implications for the age of Kupferschiefer mineralization. Economic Geology, 94: 261-272.

Bechtel, A., Gratzer, R., Püttmann, W., Oszczepalski, S., 2001a Variable alteration of organic matter in relation to metal zoning at the Rote Fäule front (Lubin-Sieroszowice mining district, SW Poland). Organic Geochemistry, 32: 377-395.
Bechtel, A., Ghazi, A.M., Elliott, W.C., Oszczepalski, S., 2001 b. The occurrences of the rare earth elements and the platinum group elements in relation to base metal zoning in the vicinity of Rote Fäule in the Kupferschiefer of Poland. Applied Geochemistry, 16: 375-386.

Bechtel, A., Gratzer, R., Püttmann, W., Oszczepalski, S., 2002. Geochemical characteristics across the oxic/anoxic interface (Rote Fäule front) within the Kupferschiefer of the Lubin-Sieroszowice mining district (SW Poland). Chemical Geology, 185: 9-31.

Blundell, D.J., Karnkowski, P., Alderton, D.H.M., Oszczepalski, S., Kucha, H., 2003. Copper mineralization of the Polish 
Kupferschiefer: a proposed basement fault-fracture system of fluid flow. Economic Geology, 98: 1487-1495.

Bouch, J.E., Hole, M.J., Trewin, N.H., Morton, A.C., 2002. Authigenic apatite in a fluvial sandstone sequence: evidence for rare-earth element mobility during diagenesis and a tool for diagenetic correlation. Journal of Sedimentary Research, 72: $59-67$.

Brugger, J., Ogierman, J., Pring, A., Waldron, H., Kolitsch, U., 2006. Origin of the secondary REE-minerals at the Paratoo copper deposit near Yunta, South Australia. Mineralogical Magazine, 70: 609-627.

Cathles, L.M. III, Oszczepalski, S., Jowett, E.C., 1993. Mass balance evaluation of the late diagenetic hypothesis for Kupferschiefer Cu mineralization in the Lubin basin of southwestern Poland. Economic Geology, 88: 948-956.

Chmielewski, A., 2014. Characteristic of relict mineralization in the western part of Radwanice Copper Field (SW part of Lubin-Sieroszowice deposit) (in Polish with English summary). Biuletyn Państwowego Instytutu Geologicznego, 458: 1-24.

Chmielewski, A., Oszczepalski, S., Speczik, S., 2015. Relict mineralization in the transition zone, Kupferschiefer series of SW Poland. In: Mineral resources in a sustainable world (eds. A-S. Andre-Mayer, M. Cathelineau, P. Muchez, E. Pirard and S. Sindern). Proceedings Volume 5: 1897-1900. GeoRessources, Universite de Lorraine.

Cruse, A.M., Lyons, T.W., Cantrell, K.J., 2000. Rare-earth element behavior in phosphates and organic-rich host shales: an example from the Upper Carboniferous of midcontinent North America. Marine authigenesis from global to microbial. SEPM Special Publication, 66: 445-453.

De Baar, H.J., German, C.R., Elderfield, H., van Gaans, P., 1988. Rare earth element distribution in anoxic waters of the Cariaco Trench. Geochimica et Cosmochimica Acta, 52: 1203-1219.

Galos, K., Nieć, M., Radwanek-Bąk, B., Smakowski, T., Szamałek, K., 2012. The mineral security of Poland within the EU and in the World. Biuletyn Państwowego Instytutu Geologicznego, 452: 43-52.

Gammons, C.H., Wood, S.A., Williams-Jones, A.E., 1996. The aqueous geochemistry of the rare earth elements and yttrium. VI. Stability of neodymium chloride complexes from 25 to $300^{\circ} \mathrm{C}$. Geochimica et Cosmochimica Acta, 60: 4615-4630.

Gammons, C.H., Wood, S.A., Jonas, J.P., Madison, J.P., 2003. Geochemistry of the rare-earth elements and uranium in the acidic Berkeley Pit Lake, Butte, Montana. Chemical Geology, 198: 269-288.

German, C.R., Holliday, B.P., Elderfield, H., 1991. Redox cycling of rare earth elements in the suboxic zone of the Black Sea. Geochimica et Cosmochimica Acta, 55: 3553-3558.

Gieré, R., 1996. Formation of rare earth minerals in hydrothermal systems. In: Rare Earth Minerals, Chemistry, Origin and Ore Deposits (eds. A.P. Jones, F. Wall and C.T. Williams): 105-150. The Mineralogical Society Series, Chapmann and Hall, London.

Goodenough, K.M., Schilling, J., Jonsson, E., Kalvig, P., Charles, N., Tuduri, J., Deady, E.A., Sadeghi, M., Schiellerup, H., Müller, A., Bertrand, G., Arvanitidis, N., Eliopoulos, D.G., Shaw, R.A., Thrane, K., Keulen, N., 2016. Europe's rare earth element resource potential: an overview of REE metallogenetic provinces and their geodynamic setting. Ore Geology Reviews, 72: 838-856.

Gosselin, D.G., Smith, M.R., Lepell, E.A., Laul, J.C., 1992. Rare earth elements in chloride-rich groundwater, Palo Duro Basin, Texas, USA. Geochimica et Cosmochimica Acta, 56: 1495-1505.

Gromet, P.L., Dymek, P.F., Haskin, L.A., Korotev, R.I., 1984. The North American Shale Composite: its composition, major and minor element characteristics. Geochimica et Cosmochimica Acta, 48: 2469-2482.

Hammer, J., Junge, F., Rösler, H.J.S., Gleisberg, B., Stiehl, G., 1990. Element and isotope geochemical investigations of the Kupferschiefer in the vicinity of "Rote Fäule", indicating copper mineralization (Sangerhausen basin, G.D.R.). Chemical Geology, 85: 345-360.
Hannigan, R.E., Sholkovitz, E.R., 2001. The development of middle rare earth element enrichments in freshwaters: weathering of phosphate minerals. Chemical Geology, 175: 495-508.

Hartmann, B.T., Gaupp, R., Oberhänsli, R., 1997. Authigenic rare earth fluorocarbonates (synchiste) in Rotliegend sandstone from the North-German Basin: constraints for REE mobility during diagenesis. Gaea Heidelbergensis, 3: 158.

Huyck, H.L.O., 1989. When is a metalliferous black shale not a black shale? U.S. Geological Survey Circular, 1058: 42-56.

Jaireth, S., Hoatson, D.M., Miezitis, Y., 2014. Geological setting and resources of the major rare-earth-element deposits in Australia. Ore Geology Reviews, 62: 72-128.

Janczyszyn, J., Loska, L., Mayer, W., Piestrzyński, A., Domańska, G., 1986. Investigation of the copper bearing shales with the use of neutron activation analysis with particular regard to noble metals, rare earth elements, uranium and thorium (in Polish with English summary). Gospodarka Surowcami Mineralnymi, 2: 483-496.

Jiang, S.Y., Chen, Y.Q., Ling, H.F., Yang, J.H., Feng, H-Z., Ni, P., 2006. Trace- and rare-earth element geochemistry and $\mathrm{Pb}-\mathrm{Pb}$ dating of black shales and intercalated Bi-Mo-PGE-Au sulphide ores in Lower Cambrian strata, Yangtze Platform, South China. Mineralium Deposita, 41: 453-467.

Johannesson, K.H., Lyons, W.B., Yelken, M.A., Gaudette, H.E., Stetzenbach, K.J., 1996. Geochemistry of rare-earth elements in hypersaline and dilute acidic natural terrestrial waters: complexation behaviour and middle rare-earth element enrichment. Chemical Geology, 133: 125-144.

Jowett, E.C., Pearce, G.W., Rydzewski, A., 1987. A Mid-Triassic paleomagnetic age of the Kupferschiefer mineralization in Poland based on a revised apparent polar wander path of Europe and Russia. Journal of Geophysical Research, 92: 581-598.

Kiersnowski, H., Peryt, T.M., Buniak, A., Mikołajewski, Z., 2010. From the intra-desert ridges to the marine carbonate island chain: middle to late Permian (Upper Rotliegend-Lower Zechstein) of the Wolsztyn-Pogorzela high, west Poland. Geological Journal, 46: 319-335.

Kucha, H., 1982. Platinum-group metals in the Zechstein copper deposits, Poland. Economic Geology, 77: 1578-1591.

Kucha, H., Pawlikowski, M., 1986. Two-brine model of the genesis of strata-bound Zechstein deposits (Kupferschiefer type), Poland. Mineralium Deposita, 25: 262-271.

Kucha, H., Przybyłowicz, W., 1999. Noble metals in organic matter and clay-organic matrices, Kupferschiefer, Poland. Economic Geology, 94: 1137-1162.

Long, K.R., Van Gosen, B.S., Foley, N.K., Cordier, D., 2012. The principal rare earth elements deposits of the United States: a summary of domestic deposits and a global perspective. In: Non-Renewable Resource Issues: Geoscientific and Societal Challenges (eds. R. Sinding-Larsen and F.-W. Wellmer): 131-155. International Year of Planet Earth, Springer.

Loukola-Ruskeeniemi, K., Lahtinen, H., 2013. Multiphase evolution in the black-shale-hosted $\mathrm{Ni}-\mathrm{Cu}-\mathrm{Zn}$-Co deposit at Talvivaara, Finland. Ore Geology Reviews, 52: 85-99.

Ma, L., Jin, L., Brantley, S.L., 2011. How mineralogy and slope aspect affect REE release and fractionation during shale weathering in the Susquehanna/Shale Hills Critical Zone Observatory. Chemical Geology, 290: 31-49.

Mayanovic, R.A., Anderson, A.J., Bassett, W.A., Chou, I.M., 2007. On the formation and structure of rare-earth element complexes in aqueous solutions under hydrothermal conditions with new data on gadolinium aqua and chloric complexes. Chemical Geology, 239: 266-283.

Mayer, W., Piestrzyński, A., 1986. Francolite from the Zechstein sediments, Rudna mine, Fore-Sudetic Monocline. Mineralogia Polonica, 17: 77-86.

Mayer, W., Janczyszyn, J., Piestrzyński, A., 1992. REE distribution patterns in Lower Zechstein sediments from Poland - preliminary report. Mineralogia Polonica, 23: 15-28.

McDonough, W.F., Sun, S.S., 1995. The composition of the Earth. Chemical Geology, 120: 223-253. 
McLennan, S.M., 1989. Rare earth elements in sedimentary rocks: influence of provenance and sedimentary processes. Reviews in Mineralogy and Geochemistry, 21: 169-200

Michalik, M., 2001. Diagenesis of the Weissliegend sandstones in the south-western margin of the Polish Rotliegend basin. Prace Mineralogiczne, 91: 1-176.

Migdisov, A., Williams-Jones, A.E., Brugger, J., Caporuscio, F.A., 2016. Hydrothermal transport, deposition, and fractionation of the REE: experimental data and thermodynamic calculations. Chemical Geology, 439: 13-42.

Mikulski, S.Z., Oszczepalski, S., Brański, P., Kozdrój, W., Markowiak, M., Kramarska, R., Chmielewski, A., Sadłowska, K., Damrat, M., 2014. Weryfikacja stanu wiedzy o mineralizacji metalami ziem rzadkich (REE) wraz z pilotażową oceną ich perspektyw złożowych w Polsce $z$ wyłaczeniem obszaru kratonu wschodnioeuropejskiego (in Polish). NAG, no. 1463/2016, PIG-PIB, Warszawa.

Milodowski, A.E., Zalasiewicz, J.A., 1991. Redistribution of rare earth elements during diagenesis of turbidite/hemipelagic mudrock sequences of Llandovery age from central Wales. Geological Society Special Publications, 57: 101-124.

Müller, N., Franke, K., Schreck, P., Hirsch, D., Kupsch, H., 2008. Georadiochemical evidence to weathering of mining residues of the Mansfeld mining district, Germany. Environmental Geology, 54: 869-877.

Olias, M., Cerón, J.C., Fernández, I., De la Rosa, J., 2005. Distribution of rare earth elements in an alluvial aquifer affected by acid mine drainage: the Guadiamar aquifer (SW Spain). Environmental Pollution, 135: 53-64.

Oszczepalski, S., 1989. Kupferschiefer in southwestern Poland: sedimentary environments, metal zoning, and ore controls. Geological Association of Canada Special Papers, 36: 571-600.

Oszczepalski, S., 1994. Oxidative alteration of the Kupferschiefer in Poland: oxide-sulphide parageneses and implications for ore-forming models. Geological Quarterly, 38 (4): 651-672.

Oszczepalski, S., 1999. Origin of the Kupferschiefer polymetallic mineralization in Poland. Mineralium Deposita, 34: 599-613.

Oszczepalski, S., Rydzewski, A., 1987. Paleogeography and sedimentary model of the Kupferschiefer in Poland. Lecture Notes in Earth Sciences, 10: 189-205.

Oszczepalski, S., Rydzewski, A., 1991. The Kupferschiefer mineralization in Poland. Zentralblatt für Geologie und Paläontologie, I: 975-999.

Oszczepalski, S., Rydzewski, A., 1997. Metallogenic Atlas of the Zechstein Copper-bearing Series in Poland. Państwowy Instytut Geologiczny - Wydawnictwo Kartograficzne Polskiej Agencji Ekologicznej SA., Warszawa.

Oszczepalski, S., Nowak, G.J., Bechtel, A., Žák, K., 2002. Evidence of oxidation of the Kupferschiefer in the Lubin-Sieroszowice deposit: implications for $\mathrm{Cu}-\mathrm{Ag}$ and $\mathrm{Au}-\mathrm{Pt}-\mathrm{Pd}$ mineralisation. Geological Quarterly, 46 (1): 1-23.

Oszczepalski, S., Speczik, S., Małecka, K., Chmielewski, A., 2016. Prospective copper resources in Poland. Gospodarka Surowcami Mineralnymi - Mineral Resources Management, 32 : 5-30.

Peryt, T.M., Geluk, M.C., Mathiesen, A., Paul, J., Smith, K., 2010. Zechstein. In: Petroleum Geological Atlas of the Southern Permian Basin Area (eds. J.C. Doornenbal and A.G. Stevenson): 123-147. EAGE Publications b.v.; Houten.

Peryt, T.M., Durakiewicz, T., Kotarba, M.J., Oszczepalski, S., Peryt, D., 2012a. Carbon isotope stratigraphy of the basal Zechstein (Lopingian) strata in Northern Poland and its global correlation. Geological Quarterly, 56 (2): 285-298.

Peryt, T.M., Raczyński, P., Peryt, D., Chłódek, K., 2012b. Upper Permian reef complex in the basinal facies of the Zechstein Limestone (Ca1), western Poland. Geological Journal, 46: 537-552.

Piasecki, W., Sverjensky, D.A., 2008. Speciation of adsorbed yttrium and rare earth elements on oxide surfaces. Geochimica et Cosmochimica Acta, 72: 3964-3979.

Pieczonka, J., 2011. Factors controlling distribution of ore minerals within copper deposit, Fore-Sudetic Monocline, SW Poland (in Polish with English summary). Wydawnictwa AGH, Kraków.
Pieczonka, J., Piestrzyński, A., Mucha, J., Głuszek, A., Kotarba, M., Więcław, D., 2008. The red-bed-type precious metal deposit in the Sieroszowice-Polkowice copper mining district, SW Poland. Annales Societatis Geologorum Poloniae, 78: 151-280.

Piestrzyński, A., Sawłowicz, Z., 1999. Exploration for Au and PGE in the Polish Zechstein copper deposits (Kupferschiefer). Journal of Geochemical Exploration, 66: 17-25.

Piestrzyński, A., Wodzicki, A., 2000. Origin of the gold deposit in the Polkowice-West Mine, Lubin-Sieroszowice Mining District, Poland. Mineralium Deposita, 35: 37-47.

Piestrzyński, A., Pieczonka, J., Speczik, S., Oszczepalski, S., Banaszak, A., 1997. Noble metals from the Kupferschiefer-type deposits, Lubin-Sieroszowice, SW Poland. In: Mineral Deposits: Resources and Exploration - Where do They Meet? (ed. H. Papunen): 563-566. Balkema, Rotterdam.

Piper, D.Z., 1974. Rare-earth elements in the sedimentary cycle: a summary. Chemical Geology, 14: 285-304.

Piper, D.Z., Bau, M., 2013. Normalized rare earth elements in water sediments, and wine: identifying sources and environmental redox conditions. American Journal of Analytical Chemistry, 4: 69-83.

Protano, G., Riccobono, F., 2002. High contents of rare earth elements (REEs) in stream waters of a Cu-Pb-Zn mining area. Environmental Pollution, 117: 499-514.

Püttmann, W., Merz, C., Speczik, S., 1989. The secondary oxidation of organic material and its influence on Kupferschiefer mineralization of southwest Poland. Applied Geochemistry, 4: 151-161.

Rudnick, R.L., Gao, S., 2003. Composition of the continental crust. In: Treatise on Geochemistry (eds. H.D. Holland and K.K. Turekian), vol. 3: 1-64, Elsevier, New York.

Rydzewski, A., 1978. Oxidated facies of the Zechstein copper-bearing shale in the Fore-Sudetic Monocline (in Polish with English abstract). Przegląd Geologiczny, 26: 102-108.

Sawłowicz, Z., 1993. Organic matter and its significance for the genesis of the copper-bearing shales (Kupferschiefer) from the Fore-Sudetic Monocline (Poland). In: Bitumens in Ore Deposits (eds. J. Parnell, H. Kucha and P. Landais): 431-446. Special Publication No 9 of the Society for Geology Applied to Mineral Deposits, Springer-Verlag.

Sawłowicz, Z., 1994. REE in shales from the Zechstein copper deposits from Poland. 9th Symposium IAGOD, Beijing: 325-326.

Sawłowicz, Z., 2013. REE and their relevance to the development of the Kupferschiefer copper deposit in Poland. Ore Geology Reviews, 55: 176-186.

Schieber, J., 1988. Redistribution of rare-earth elements during diagenesis of carbonate rocks from the Mid-Proterozoic Newland Formation, Montana, U.S.A. Chemical Geology, 69: 111-126.

Shields, G., Stille, P., 2001. Diagenetic constraints on the use of cerium anomalies as paleoseawater redox proxies: an isotopic and REE study of Cambrian phosphorites. Chemical Geology, 175: 29-48.

Sholkovitz, E.R., 1995. The aquatic geochemistry of rare earth elements in rivers and estuaries. Aquatic Geochemistry, 1: 1-43.

SimandI, G.J., 2014. Geology and market-dependent significance of rare earth element resources. Mineralium Deposita, 49: 889-904.

Speczik, S., Püttmann, W., 1987. Origin of Kupferschiefer mineralization as suggested by coal petrology and organic geochemical studies. Acta Geologica Polonica, 37: 167-187.

Speczik, S., Oszczepalski, S., Nowak, G.J., Grotek, I., Niczyporuk, K., 2003. Organic matter alteration trends in the Polish Kupferschiefer: ore genetic implications. In: Mineral Exploration and Sustainable Development (eds. D.G. Eliopoulos et al.): 853-856. Millpress, Rotterdam, Netherlands.

Stille, P., Clauer, N., 1994. The process of glauconitization: chemical and isotopic evidence. Contributions to Mineralogy and $\mathrm{Pe}$ trology, 117: 253-262.

Van Wees, J.-D., Stephenson, R.A., Ziegler, P.A., Bayer, U., McCann, T., Dadlez, R., Gaupp, R., Narkiewicz, M., Bitzer, F., Scheck, M., 2000. On the origin of the Southern Permian Basin, Central Europe. Marine and Petroleum Geology, 17: 43-59. 
Verplanck, P.L., Nordstrom, D.K., Taylor, H.E., Kimball, B.A., 2004. Rare earth element partitioning between hydrous ferric oxides and acid mine water during iron oxidation. Applied Geochemistry, 19: 1339-1354.

Welch, S.A., Christy, A.G., Isaacson, L., Kirste, D., 2009. Mineralogical control of rare earth elements in acid sulfate soils. Geochimica et Cosmochimica Acta, 73: 44-64.

Weng, Z., Jowitt, S.M., Mudd, G.M., Haque, N., 2015. A detailed assessment of global Rare Earth Element resources: opportunities and challenges. Economic Geology, 110: 1925-1952.

Wodzicki, A., Piestrzyński, A., 1994. An ore genetic model for the Lubin-Sieroszowice mining district, Poland. Mineralium Deposita, 29: 30-43.

Wood, S.A., 2003. The geochemistry of rare-earth elements and ytrium in geothermal waters. SEG Special Publication, 10: 133-158.
Worrall, F., Pearson, D.G., 2001. Water-rock interaction in an acidic mine discharge as indicated by rare earth element patterns. Geochimica et Cosmochimica Acta, 65: 3027-3040.

Zanin, Y.N., Eder, V.G., Zamirailova, A.G., Krasovchikov, V.O., 2010. Models of the REE distribution in the black shale Bazhenov Formation of the West Siberian marine basin, Russia. Chemie der Erde, 70: 363-376.

Zhao, F., Cong, Z., Sun, H., Ren, D., 2007. The geochemistry of rare earth elements (REE) in acid mine drainage from the Sitai coal mine, Shanxi Province, North China. International Journal of Coal Geology, 70: 184-192.

Zwingmann, H., Clauer, N., Gaupp, R., 1999. Structure-related geochemical (REE) and isotopic (K-Ar, Rb-Sr, $\left.\delta^{18} \mathrm{O}\right)$ characteristics of clay minerals from Rotliegend sandstone reservoirs (Permian, northern Germany). Geochimica et Cosmochimica Acta, 63: 2805-2823. 